This is a self-archived version of an original article. This version may differ from the original in pagination and typographic details.

Author(s): Parkkonen, Jouni; Paulin, Frédéric

Title: Counting and equidistribution in Heisenberg groups

Year: 2017

Version: Accepted version (Final draft)

Copyright: @ Springer-Verlag Berlin Heidelberg 2016

Rights: In Copyright

Rights url: http://rightsstatements.org/page/lnC/1.0/?language=en

Please cite the original version:

Parkkonen, J., \& Paulin, F. (2017). Counting and equidistribution in Heisenberg groups. Mathematische Annalen, 367(1), 81-119. https://doi.org/10.1007/s00208-015-1350-5 


\title{
Counting and equidistribution in Heisenberg groups
}

\author{
Jouni Parkkonen Frédéric Paulin
}

February 13, 2019

\begin{abstract}
We strongly develop the relationship between complex hyperbolic geometry and arithmetic counting or equidistribution applications, that arises from the action of arithmetic groups on complex hyperbolic spaces, especially in dimension 2 . We prove a Mertens' formula for the integer points over a quadratic imaginary number fields $K$ in the light cone of Hermitian forms, as well as an equidistribution theorem of the set of rational points over $K$ in Heisenberg groups. We give a counting formula for the cubic points over $K$ in the complex projective plane whose Galois conjugates are orthogonal and isotropic for a given Hermitian form over $K$, and a counting and equidistribution result for arithmetic chains in the Heisenberg group when their Cygan diameter tends to 0.1
\end{abstract}

\section{Introduction}

The aim of this paper is to give original asymptotic counting and equidistribution results with error terms of arithmetically defined points or circles in nilmanifolds covered by the Heisenberg groups. We refer for instance to [Bre, BF, GT, Kim, BeQ] or [EiW, Chap. 10] for other types of results.

One of our main results (see Section 7) is an asymptotic counting result of arithmetic chains in hyperspherical geometry. Let $q$ be the Hermitian form $-z_{0} \overline{z_{2}}-z_{2} \overline{z_{0}}+\left|z_{1}\right|^{2}$ on $\mathbb{C}^{3}$. Its isotropic locus in the complex projective plane $\mathbb{P}_{2}(\mathbb{C})$ is called by Poincaré the hypersphere [Poi]. With [1:0:0] removed, the hypersphere identifies with the 3dimensional Heisenberg group Heis ${ }_{3}$ (central extension of $\mathbb{C}$ by $\mathbb{R}$ ), and it carries a natural distance $d_{\mathrm{Cyg}}$, called Cygan's (sometimes Korányi's) distance. Recall that a chain, as introduced by von Staudt and developped in particular by E. Cartan, see for instance [Car] and [Gol, §4.3], is a nontrivial intersection with the hypersphere of a complex projective line. A chain is either a fiber of the canonical morphism Heis $3 \rightarrow \mathbb{C}$ or an ellipse whose projection by this map is a circle. Let $K$ be an imaginary quadratic number field, and let $\mathscr{O}_{K}$ be its ring of integers. We say that a chain $C_{0}$ is arithmetic (over $K$ ) if the orbit of some point in $C_{0}$ under the stabiliser of $C_{0}$ in the arithmetic lattice $\operatorname{PSU}_{q}\left(\mathscr{O}_{K}\right)$ is dense in $C_{0}$. The stabiliser $\operatorname{PSU}_{q}\left(\mathscr{O}_{K}\right)_{\infty}$ of $[1: 0: 0]$ in $\operatorname{PSU}_{q}\left(\mathscr{O}_{K}\right)$ preserves the diameters of the chains for $d_{\mathrm{Cyg}}$. The picture below shows an orbit of arithmetic chains under the arithmetic lattice $\operatorname{PSU}_{q}(\mathbb{Z}[i])$.

\footnotetext{
${ }^{1}$ Keywords: counting, equidistribution, Mertens formula, Heisenberg group, Cygan distance, subRiemannian geometry, common perpendicular, complex hyperbolic geometry, chain, cubic point. AMS codes: $11 \mathrm{E} 39,11 \mathrm{~F} 06,11 \mathrm{~N} 45,20 \mathrm{G} 20,53 \mathrm{C} 17,53 \mathrm{C} 22,53 \mathrm{C} 55$
} 


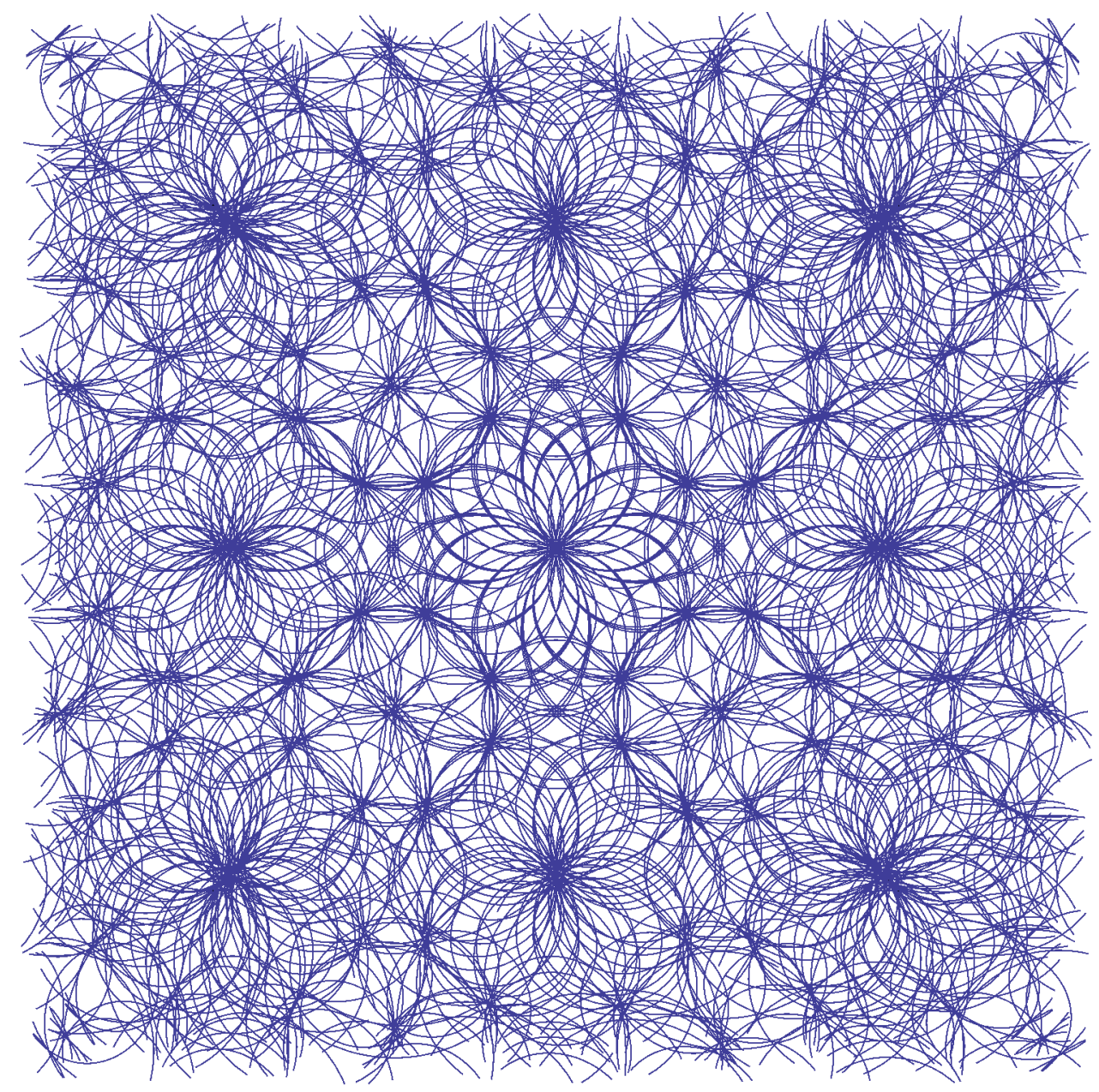

Theorem 1 Let $C_{0}$ be an arithmetic chain in the hypersphere. There exists a constant $\kappa>0$ and an explicit constant $c>0$ such that, as $\epsilon \rightarrow 0$, the number of chains modulo $\operatorname{PSU}_{q}\left(\mathscr{O}_{K}\right)_{\infty}$ in the $\operatorname{PSU}_{q}\left(\mathscr{O}_{K}\right)$-orbit of $C_{0}$, with diameter at least $\epsilon$, is $c \epsilon^{-4}\left(1+\mathrm{O}\left(\epsilon^{\kappa}\right)\right)$.

We will also prove that the centers of the finite arithmetic chains equidistribute in the hypersphere.

An analogous method allows us in Section 6 to prove the following counting result of some arithmetic points in the complex projective plane $\mathbb{P}_{2}(\mathbb{C})$. Let $z_{0} \in \mathbb{P}_{2}(\mathbb{C})$ be a cubic point over $K$, whose Galois conjugates $z_{0}^{\prime}, z_{0}^{\prime \prime}$ over $K$ are isotropic and orthogonal to $z_{0}$ for the Hermitian form $q$. The arithmetic lattice $\operatorname{PSU}_{q}\left(\mathscr{O}_{K}\right)$ acts with infinitely many orbits on the set of such points. The inverse of a slightly modified version $d_{\text {Cyg }}^{\prime \prime}$ (see Section 3 for precise definitions) of the Cygan distance between the two isotropic conjugates over $\bar{K}$ is a natural positive complexity $c$ on the orbit of $z_{0}$ under $\operatorname{PSU}_{q}\left(\mathscr{O}_{K}\right)$, which is invariant under $\operatorname{PSU}_{q}\left(\mathscr{O}_{K}\right)_{\infty}$.

Theorem 2 There exists a constant $\kappa>0$ and an explicit constant $C>0$ such that, as $s \rightarrow+\infty$,

$$
\operatorname{Card}\left\{z \in \operatorname{PSU}_{q}\left(\mathscr{O}_{K}\right)_{\infty} \backslash \operatorname{PSU}_{q}\left(\mathscr{O}_{K}\right) \cdot z_{0}: c(z) \leq s\right\}=C s^{4}\left(1+\mathrm{O}\left(s^{-\kappa}\right)\right) .
$$


We refer to Section 6 for a more precise and more general statement, valid for instance for the congruence subgroups of $\operatorname{PSU}_{q}\left(\mathscr{O}_{K}\right)$.

The main tools of this paper, described in Section 2, are a geometric counting result for the common perpendiculars between two properly immersed closed convex subsets, and an equidistribution result of their initial and terminal tangent vectors, valid in general pinched negative curvature [PaP7]. See also OhS1, OhS2 for related counting and equidistribution results in real hyperbolic spaces, $\mathrm{Kim}$ in negatively curved symmetric spaces, [PaP5] for more geometric applications, and $\mathrm{PaP} 4]$ for arithmetic applications using real hyperbolic spaces.

We recall the necessary geometric background on the complex hyperbolic spaces in Section 3. Section 4 is devoted to the computation of the measure theoretic constants that appear in the geometric counting and equidistribution result in the special case of the complex hyperbolic spaces, as was done in [PaP6, §7] in the case of the real hyperbolic spaces. In particular, these computations allow to obtain the following geometric counting result (see Corollary 13 for a more general version).

Theorem 3 Let $\Gamma$ be a discrete group of isometries of the complex hyperbolic $n$-space $\mathbb{H}_{\mathbb{C}}^{n}$ such that the orbifold $M=\Gamma \backslash \mathbb{H}_{\mathbb{C}}^{n}$ has finite volume. Let $D^{-}$be a horoball in $\mathbb{H}_{\mathbb{C}}^{n}$ centred at a parabolic fixed point of $\Gamma$ and let $D^{+}$be a complex geodesic line in $\mathbb{H}_{\mathbb{C}}^{n}$ whose quotient under its stabiliser $\Gamma_{D^{+}}$in $\Gamma$ has finite volume, and let $m^{+}$be the cardinality of the pointwise stabiliser of $D^{+}$in $\Gamma$. Then the number (counted with multiplicities) of the common perpendiculars of length at most $t$ between the images of $D^{-}$and $D^{+}$in $M$ is equivalent as $t \rightarrow+\infty$ to

$$
\frac{4(n-1)}{(2 n-1) \pi} \frac{\operatorname{Vol}\left(\Gamma_{D^{-}} \backslash D^{-}\right) \operatorname{Vol}\left(\Gamma_{D^{+}} \backslash D^{+}\right)}{m^{+} \operatorname{Vol}(M)} e^{2 n t} .
$$

In order to motivate our next results, let us state in an appropriate way the classical result, known as Mertens' formula, describing the asymptotic behaviour of the average order of Euler's function, or equivalently the asymptotic counting of Farey fractions, and its related equidistribution result of Farey fractions in the group $\mathbb{R}$. The additive group $\mathbb{Z}$ acts on $\mathbb{Z} \times \mathbb{Z}$ by horizontal shears (transvections): $k \cdot(u, v)=(u+k v, v)$. Then

$$
\text { Card } \mathbb{Z} \backslash\{(u, v) \in \mathbb{Z} \times \mathbb{Z}:(u, v)=1,|v| \leq s\}=\frac{6}{\pi^{2}} s+\mathrm{O}\left(s^{1-\kappa}\right)
$$

for some $\kappa>0$ (see for example [HaW, Thm. 330], a better error term is due to Walfisz Wal]). Furthermore, with $\Delta_{x}$ the unit Dirac mass at $x$, as $s \rightarrow+\infty$,

$$
\frac{\pi^{2}}{6 s} \sum_{(u, v)=1,|v| \leq s} \Delta_{\frac{u}{v}} \stackrel{*}{\rightarrow} \operatorname{Leb}_{\mathbb{R}} .
$$

Our next result is an analog of Mertens' formula for Heisenberg groups. Let tr, $\mathrm{n}$ be the (absolute) trace and norm of $K$, and let $\langle a, b, c\rangle$ be the ideal of $\mathscr{O}_{K}$ generated by $a, b, c \in \mathscr{O}_{K}$. The nilpotent group

$$
\operatorname{Heis}_{3}\left(\mathscr{O}_{K}\right)=\left\{\left(w_{0}, w\right) \in \mathscr{O}_{K} \times \mathscr{O}_{K}: \operatorname{tr}\left(w_{0}\right)=\mathrm{n}(w)\right\}
$$

with law

$$
\left(w_{0}, w\right)\left(w_{0}^{\prime}, w^{\prime}\right)=\left(w_{0}+w_{0}^{\prime}+w^{\prime} \bar{w}, w+w^{\prime}\right)
$$


acts on $\mathscr{O}_{K} \times \mathscr{O}_{K} \times \mathscr{O}_{K}$ by the shears

$$
\left(w_{0}, w\right)(a, \alpha, c)=\left(a+\bar{w} \alpha+w_{0} c, \alpha+w c, c\right) .
$$

Theorem 4 There exists $\kappa>0$ such that, as $s \rightarrow+\infty$,

$$
\begin{aligned}
& \text { Card } \operatorname{Heis}_{3}\left(\mathscr{O}_{K}\right) \backslash\left\{(a, \alpha, c) \in \mathscr{O}_{K} \times \mathscr{O}_{K} \times \mathscr{O}_{K}: \begin{array}{c}
\langle a, \alpha, c\rangle=\mathscr{O}_{K}, \\
\operatorname{tr}(a \bar{c})=\mathrm{n}(\alpha), \mathrm{n}(c) \leq s
\end{array}\right\} \\
= & \frac{\zeta(3)}{2 \pi\left|D_{K}\right|^{\frac{1}{2}} \zeta_{K}(3)} s^{2}+\mathrm{O}\left(s^{2-\kappa}\right),
\end{aligned}
$$

where $D_{K}$ is the discriminant and $\zeta_{K}$ Dedekind's zeta function of $K$.

The 3-dimensional Heisenberg group

$$
\operatorname{Heis}_{3}=\left\{\left(w_{0}, w\right) \in \mathbb{C} \times \mathbb{C}: 2 \operatorname{Re} w_{0}=|w|^{2}\right\},
$$

with the group law defined by Equation (1) is the Lie group of $\mathbb{R}$-points of a $\mathbb{Q}$-group whose group of $\mathbb{Q}$-points is $\mathrm{Heis}_{3} \cap(K \times K)$. We endow it with its Haar measure

$$
d \operatorname{Haar}_{\mathrm{Heis}_{3}}\left(w_{0}, w\right)=d\left(\operatorname{Im} w_{0}\right) d(\operatorname{Re} w) d(\operatorname{Im} w) .
$$

Theorem 4 is a counting result of rational points $\left(\frac{a}{c}, \frac{\alpha}{c}\right)$ (analogous to Farey fractions) in $\mathrm{Heis}_{3}$, and the following result is a related equidistribution theorem.

Theorem 5 As $s \rightarrow+\infty$, we have

$$
\frac{\pi\left|D_{K}\right|^{\frac{3}{2}} \zeta_{K}(3)}{\zeta(3)} s^{-2} \sum_{\substack{(a, \alpha, c) \in \mathscr{O}_{K} \times \mathscr{O}_{K} \times \mathscr{O}_{K}, 0<\mathrm{n}(c) \leq s \\ \operatorname{tr}(a \bar{c})=\mathrm{n}(\alpha),\langle a, \alpha, c\rangle=\mathscr{O}_{K}}} \Delta_{\left(\frac{a}{c}, \frac{\alpha}{c}\right)} \stackrel{*}{*} \mathrm{Haar}_{\mathrm{Heis}_{3}} .
$$

Theorems 4 and 5 being of an arithmetic nature without any reference to the geometry used in their proofs, can certainly be proven using techniques from analytic number theory, and such a direct approach can produce somewhat more precise results. We refer to Theorems 14 and 15 in Section 5 for more general results with added congruence properties, and to Remark 18 for counting and equidistribution results in higher dimensional Heisenberg groups.

Acknowledgement: The first author thanks the Université de Paris-Sud (Orsay) for a visit of a month and a half which allowed an important part of the writing of this paper, under the financial support of the ERC grant GADA 208091. We thank Y. Benoist and L. Clozel for their help with Proposition 20.

\section{Geometric counting and equidistribution}

In this Section, we briefly review a simplified version of the geometric counting and equidistribution results proved in [PaP7], whose arithmetic applications will be considered in the main part of this paper (see also [PaP6] for a review of related references).

Let $\widetilde{M}$ be a negatively curved rank one symmetric space (see [PaP7, §2] for a more general setting). In other words, $\widetilde{M}$ is a hyperbolic space $\mathbb{H}_{\mathbb{F}}^{n}$ where $\mathbb{F}$ is the set $\mathbb{R}$ of real 
numbers, $\mathbb{C}$ of complex numbers, $\mathbb{H}$ of Hamilton's quaternions, or $\mathbb{O}$ of octonions, and $n \geq 2$, with $n=2$ if $\mathbb{K}=\mathbb{O}$ see for instance [Mos, Par3]). We will normalise them so that their maximal sectional curvature is -1 .

Let $\Gamma$ be a discrete nonelementary group of isometries of $\widetilde{M}$ and let $M=\Gamma \backslash \widetilde{M}$ be the quotient orbifold. Let $D^{-}$and $D^{+}$be nonempty proper closed convex subsets of $\widetilde{M}$, such that the families $\left(\gamma D^{-}\right)_{\gamma \in \Gamma}$ and $\left(\gamma D^{+}\right)_{\gamma \in \Gamma}$ are locally finite in $\widetilde{M}$ (see [PaP7, §3.3] for more general families). Let $\Gamma_{D^{-}}$and $\Gamma_{D^{+}}$be the stabilisers in $\Gamma$ of the subsets $D^{-}$and $D^{+}$, respectively.

We denote by $\partial_{\infty} \widetilde{M}$ the boundary at infinity of $\widetilde{M}$, by $\Lambda \Gamma$ the limit set of $\Gamma$ and by $(\xi, x, y) \mapsto \beta_{\xi}(x, y)$ the Busemann function on $\partial_{\infty} \widetilde{M} \times \widetilde{M} \times \widetilde{M}$ (see for instance [BrH] ). For every $v \in T^{1} \widetilde{M}$, let $\pi(v) \in \widetilde{M}$ be its origin, and let $v_{-}, v_{+}$be the points at infinity of the geodesic line defined by $v$. We denote by $\partial_{ \pm}^{1} D^{\mp}$ the outer/inner unit normal bundle of $\partial D^{\mp}$, that is, the set of $v \in T^{1} \widetilde{M}$ such that $\pi(v) \in \partial D^{\mp}$ and the closest point projection on $D^{\mp}$ of $v_{ \pm} \in \partial_{\infty} \widetilde{M}-\partial_{\infty} D^{\mp}$ is $\pi(v)$. For all $\gamma, \gamma^{\prime}$ in $\Gamma$, the convex sets $\gamma D^{-}$and $\gamma^{\prime} D^{+}$ have a common perpendicular if and only if their closures $\overline{\gamma D^{-}}$and $\overline{\gamma^{\prime} D^{+}}$in $\widetilde{M} \cup \partial_{\infty} \widetilde{M}$ do not intersect. We denote by $\alpha_{\gamma, \gamma^{\prime}}$ this common perpendicular (starting from $\gamma D^{-}$at time $t=0)$ and by $\ell\left(\alpha_{\gamma, \gamma^{\prime}}\right)$ its length. The multiplicity of $\alpha_{\gamma, \gamma^{\prime}}$ is

$$
m_{\gamma, \gamma^{\prime}}=\frac{1}{\operatorname{Card}\left(\gamma \Gamma_{D^{-}} \gamma^{-1} \cap \gamma^{\prime} \Gamma_{D^{+}} \gamma^{\prime-1}\right)},
$$

which equals 1 when $\Gamma$ acts freely on $T^{1} \widetilde{M}$ (for instance when $\Gamma$ is torsion-free). Let

$$
\mathscr{N}_{D^{-}, D^{+}}(t)=\sum_{\left(\gamma, \gamma^{\prime}\right) \in \Gamma \backslash\left(\left(\Gamma / \Gamma_{D^{-}}\right) \times\left(\Gamma / \Gamma_{D^{+}}\right)\right): \overline{\gamma D^{-}} \cap \overline{\gamma^{\prime} D^{+}}=\emptyset, \ell\left(\alpha_{\gamma, \gamma^{\prime}}\right) \leq t} m_{\gamma, \gamma^{\prime}},
$$

where $\Gamma$ acts diagonally on $\Gamma \times \Gamma$. When $\Gamma$ has no torsion, $\mathscr{N}_{D^{-}, D^{+}}(t)$ is the number (with multiplicities coming from the fact that $\Gamma_{D^{ \pm}} \backslash D^{ \pm}$is not assumed to be embedded in $M$ ) of the common perpendiculars of length at most $t$ between the images of $D^{-}$and $D^{+}$in $M$. We refer to [PaP7, §4] for the use of Hölder potentials on $T^{1} \widetilde{M}$ to modify this counting function by adding weights, which could be useful for some further arithmetic applications.

Recall the following notions (see for instance [Rob]). Fix a basepoint $x_{0} \in \widetilde{M}$. The critical exponent of $\Gamma$ is

$$
\delta_{\Gamma}=\limsup _{n \rightarrow+\infty} \frac{1}{n} \ln \operatorname{Card}\left\{\gamma \in \Gamma: d\left(x_{0}, \gamma x_{0}\right) \leq n\right\},
$$

which is positive, finite and independent of $x_{0} \in \widetilde{M}$. Let $\left(\mu_{x}\right)_{x \in \widetilde{M}}$ be a Patterson density for $\Gamma$, that is a family $\left(\mu_{x}\right)_{x \in \widetilde{M}}$ of nonzero finite measures on $\partial_{\infty} \widetilde{M}$ whose support is $\Lambda \Gamma$, such that $\gamma_{*} \mu_{x}=\mu_{\gamma x}$ and

$$
\frac{d \mu_{x}}{d \mu_{y}}(\xi)=e^{-\delta_{\Gamma} \beta_{\xi}(x, y)}
$$

for all $\gamma \in \Gamma, x, y \in \widetilde{M}$ and $\xi \in \partial_{\infty} \widetilde{M}$.

The Bowen-Margulis measure $\widetilde{m}_{\mathrm{BM}}$ for $\Gamma$ on $T^{1} \widetilde{M}$ is defined, using Hopf's parametrisation $v \mapsto\left(v_{-}, v_{+}, \beta_{v_{+}}\left(x_{0}, \pi(v)\right)\right)$ from $T^{1} \widetilde{M}$ into $\partial_{\infty} \widetilde{M} \times \partial_{\infty} \widetilde{M} \times \mathbb{R}$, by

$$
d \widetilde{m}_{\mathrm{BM}}(v)=e^{-\delta_{\Gamma}\left(\beta_{v_{-}}\left(\pi(v), x_{0}\right)+\beta_{v_{+}}\left(\pi(v), x_{0}\right)\right)} d \mu_{x_{0}}\left(v_{-}\right) d \mu_{x_{0}}\left(v_{+}\right) d t .
$$


Note that in the right hand side of this equation, $\pi(v)$ may be replaced by any point $x^{\prime}$ on the geodesic line defined by $v$, since $\beta_{v_{-}}\left(\pi(v), x^{\prime}\right)+\beta_{v_{+}}\left(\pi(v), x^{\prime}\right)=0$. We will use this elementary observation in the proof of Lemma 12(ii). The measure $\widetilde{m}_{\mathrm{BM}}$ is nonzero, independent of $x_{0} \in \widetilde{M}$, is invariant under the geodesic flow, the antipodal map $v \mapsto-v$ and the action of $\Gamma$. Thus, it defines a nonzero measure $m_{\mathrm{BM}}$ on $T^{1} M$ which is invariant under the geodesic flow of $M$ and the antipodal map, called the Bowen-Margulis measure on $M=\Gamma \backslash \widetilde{M}$. When $m_{\mathrm{BM}}$ is finite (for instance when $M$ has finite volume or when $\Gamma$ is geometrically finite), denoting the total mass of a measure $m$ by $\|m\|$, the probability measure $\frac{m_{\mathrm{BM}}}{\left\|m_{\mathrm{BM}}\right\|}$ is then uniquely defined, and is the unique probability measure of maximal entropy for the geodesic flow (see [OP]).

Using the endpoint homeomorphism $v \mapsto v_{+}$from $\partial_{+}^{1} D^{-}$to $\partial_{\infty} \widetilde{M}-\partial_{\infty} D^{-}$, we defined in [PaP3] (generalising the definition of Oh and Shah [OhS1, §1.2] when $D^{-}$is a horoball or a totally geodesic subspace in $\left.\mathbb{H}_{\mathbb{R}}^{n}\right)$ the skinning measure $\widetilde{\sigma}_{D^{-}}$of $\Gamma$ on $\partial_{+}^{1} D^{-}$, by

$$
d \widetilde{\sigma}_{D^{-}}(v)=e^{-\delta_{\Gamma} \beta_{v_{+}}\left(\pi(v), x_{0}\right)} d \mu_{x_{0}}\left(v_{+}\right) .
$$

The measure $\widetilde{\sigma}_{D^{-}}$is independent of $x_{0} \in \widetilde{M}$, is nonzero if $\Lambda \Gamma$ is not contained in $\partial_{\infty} D^{-}$, and satisfies $\widetilde{\sigma}_{\gamma D^{-}}=\gamma_{*} \widetilde{\sigma}_{D^{-}}$for every $\gamma \in \Gamma$. Since the family $\left(\gamma D^{-}\right)_{\gamma \in \Gamma}$ is locally finite in $\widetilde{M}$, the measure $\sum_{\gamma \in \Gamma / \Gamma_{D^{-}}} \gamma_{*} \widetilde{\sigma}_{D^{-}}$is a well defined $\Gamma$-invariant locally finite (nonnegative Borel) measure on $T^{1} \widetilde{M}$. Hence, it induces a locally finite measure $\sigma_{D^{-}}$on $T^{1} M=\Gamma \backslash T^{1} \widetilde{M}$, called the skinning measure of $D^{-}$in $T^{1} M$. We refer to [OhS2, §5] and [PaP3, Theo. 9] for finiteness criteria of the skinning measure $\sigma_{D^{-}}$, in particular satisfied when $M$ has finite volume and if either $D^{-}$is a horoball centred at a parabolic fixed point of $\Gamma$ or if $D^{-}$is a totally geodesic subspace.

Remark 6 Let $\mathscr{H}$ be a horoball in $\widetilde{M}$ centred at $\xi$, and let $\rho$ be the geodesic ray starting from any point in $\partial \mathscr{H}$ and converging to $\xi$. Then (see for instance [HeP1, §2.3]) the weak-star limit

$$
\mu_{\xi}=\lim _{t \rightarrow+\infty} e^{\delta_{\Gamma} t} \mu_{\rho(t)}
$$

exists, defines a measure on $\partial_{\infty} \widetilde{M}-\{\xi\}$ which is invariant under the elements of $\Gamma$ preserving $\mathscr{H}$. The limit measure satisfies

$$
\frac{d \mu_{x}}{d \mu_{\xi}}(\eta)=e^{-\delta_{\Gamma} \beta_{\eta}\left(x, x_{\mathscr{H}}, \eta\right)}
$$

for all $x \in \widetilde{M}, \eta \in \partial_{\infty} \widetilde{M}-\{\xi\}$, where $x_{\mathscr{H}, \eta}$ is the intersection with $\partial \mathscr{H}$ of the geodesic line from $\eta$ to $\xi$. Take $x_{0}=\rho(t)$ and let $t$ go to $+\infty$ in the definition of the Bowen-Margulis measure and the skinning measures. Then, for every $v \in T^{1} \widetilde{M}$ such that $v_{ \pm} \neq \xi$, we have

$$
d \widetilde{m}_{\mathrm{BM}}(v)=e^{-\delta_{\Gamma}\left(\beta_{v_{-}}\left(\pi(v), x \mathscr{H}, v_{-}\right)+\beta_{v_{+}}\left(\pi(v), x \mathscr{H}, v_{+}\right)\right)} d \mu_{\xi}\left(v_{-}\right) d \mu_{\xi}\left(v_{+}\right) d t .
$$

Furthermore, for every $v \in \partial_{+}^{1} \mathscr{H}$, we have

$$
d \widetilde{\sigma}_{\mathscr{H}}(v)=d \mu_{\xi}\left(v_{+}\right)
$$

since $\beta_{v_{+}}(\pi(v), \rho(t))=-t+\mathrm{o}(1)$ as $t \rightarrow+\infty$. 
The following result is a special case of [PaP7, Coro. 20, 21, Theo. 28] (we prove that the pairs of the initial/terminal tangent vectors of the common perpendiculars equidistribute in the product of the outer/inner tangent bundles of $D^{-} / D^{+}$). We refer to [PaP6] for a review of the particular cases known before [PaP7] (due to Huber, Margulis, Herrmann, Cosentino, Roblin, Oh-Shah, Martin-McKee-Wambach, Pollicott, and the authors for instance).

For all $t \geq 0$ and $x \in \partial D^{-}$, let

$$
m_{t}(x)=\sum_{\gamma \in \Gamma / \Gamma_{D^{+}}: \overline{D^{-}} \cap \overline{\gamma D^{+}}=\emptyset, \alpha_{e, \gamma}(0)=x, \ell\left(\alpha_{e, \gamma}\right) \leq t} m_{e, \gamma}
$$

be the multiplicity of $x$ as the origin of common perpendiculars with length at most $t$ from $D^{-}$to the elements of the $\Gamma$-orbit of $D^{+}$. We denote by $\Delta_{x}$ the unit Dirac mass at a point $x$.

Theorem 7 Let $\widetilde{M}, \Gamma, D^{-}, D^{+}$be as above. Assume that the measures $m_{\mathrm{BM}}, \sigma_{D^{-}}, \sigma_{D^{+}}$ are nonzero and finite. Then

$$
\mathscr{N}_{D^{-}, D^{+}}(t) \sim \frac{\left\|\sigma_{D^{-}}\right\|\left\|\sigma_{D^{+}}\right\|}{\delta_{\Gamma}\left\|m_{\mathrm{BM}}\right\|} e^{\delta_{\Gamma} t}
$$

as $t \rightarrow+\infty$. If $\Gamma$ is arithmetic or if $M$ is compact, then the error term is $\mathrm{O}\left(e^{\left(\delta_{\Gamma}-\kappa\right) t}\right)$ for some $\kappa>0$. Furthermore, the origins of the common perpendiculars equidistribute in the boundary of $D^{-}$:

$$
\lim _{t \rightarrow+\infty} \frac{\delta_{\Gamma}\left\|m_{\mathrm{BM}}\right\|}{\left\|\sigma_{D^{-}}\right\|\left\|\sigma_{D^{+}}\right\|} e^{-\delta_{\Gamma} t} \sum_{x \in \partial D^{-}} m_{t}(x) \Delta_{x}=\frac{\pi_{*} \widetilde{\sigma}_{D^{-}}}{\left\|\sigma_{D^{-}}\right\|}
$$

for the weak-star convergence of measures on the locally compact space $T^{1} \widetilde{M}$.

When $\partial D^{-}$is smooth, for smooth functions $\psi$ with compact support on $\partial D^{-}$, there is an error term in the equidistribution claim of Theorem 7 when the measures on both sides are evaluated on $\psi$, of the form $\mathrm{O}\left(e^{-\kappa t}\|\psi\|_{\ell}\right)$ where $\kappa>0$ and $\|\psi\|_{\ell}$ is the Sobolev norm of $\psi$ for some $\ell \in \mathbb{N}$, as proved in [PaP7, Theo. 28].

When $M$ has finite volume, the Bowen-Margulis measure $m_{\mathrm{BM}}$ coincides up to a multiplicative constant with the Liouville measure on $T^{1} M$, and the skinning measures of points, horoballs and totally geodesic subspaces $D^{ \pm}$coincide with the (homogeneous) Riemannian measures on $\partial_{ \pm}^{1} D^{\mp}$ induced by the (Sasaki's) Riemannian metric of $T^{1} \widetilde{M}$. In Section 4 , we explicit some of these proportionality constants when $\widetilde{M}$ is a complex hyperbolic space (see [PaP6, §7] for the real hyperbolic case).

\section{Complex hyperbolic geometry}

In this Section, we recall some background on the complex hyperbolic spaces, as mostly contained in Gol], and, unless otherwise stated, we will follow the conventions therein. For all $w, w^{\prime}$ in $\mathbb{C}^{n-1}$, we denote by $w \cdot \overline{w^{\prime}}=\sum_{i=1}^{n-1} w_{i} \overline{w_{i}^{\prime}}$ their standard Hermitian product, and we denote $|w|^{2}=w \cdot \bar{w}$. Recall that for every $n \geq 1$, the Siegel domain model of the complex hyperbolic $n$-space $\mathbb{H}_{\mathbb{C}}^{n}$ is

$$
\left\{\left(w_{0}, w\right) \in \mathbb{C} \times \mathbb{C}^{n-1}: 2 \operatorname{Re} w_{0}-|w|^{2}>0\right\},
$$


endowed with the Riemannian metric

$$
d s_{\mathbb{H}_{\mathbb{C}}^{n}}^{2}=\frac{1}{\left(2 \operatorname{Re} w_{0}-|w|^{2}\right)^{2}}\left(\left(d w_{0}-d w \cdot \bar{w}\right)\left(\overline{d w_{0}}-w \cdot \overline{d w}\right)+\left(2 \operatorname{Re} w_{0}-|w|^{2}\right) d w \cdot \overline{d w}\right) .
$$

In accordance with Section 2, this metric is normalised so that its sectional curvatures are in $[-4,-1]$, instead of in $\left[-1,-\frac{1}{4}\right]$ as in Gol] and [Par4]. Its boundary at infinity is

$$
\partial_{\infty} \mathbb{H}_{\mathbb{C}}^{n}=\left\{\left(w_{0}, w\right) \in \mathbb{C} \times \mathbb{C}^{n-1}: 2 \operatorname{Re} w_{0}-|w|^{2}=0\right\} \cup\{\infty\} .
$$

A complex geodesic line in $\mathbb{H}_{\mathbb{C}}^{n}$ is the image by an isometry of $\mathbb{H}_{\mathbb{C}}^{n}$ of the intersection of $\mathbb{H}_{\mathbb{C}}^{n}$ with the complex line $\mathbb{C} \times\{0\}$; with our normalisation of the metric, a complex line has constant sectional curvature -4 . The boundary at infinity of a complex geodesic line is a topological circle, called a chain (see Section 7 for more informations). A chain is finite if it does not contain $\infty$.

Let $q$ be the nondegenerate Hermitian form $-z_{0} \overline{z_{n}}-z_{n} \overline{z_{0}}+|z|^{2}$ of signature $(1, n)$ on $\mathbb{C} \times \mathbb{C}^{n-1} \times \mathbb{C}$ with coordinates $\left(z_{0}, z, z_{n}\right)$. This is not the form considered in Gol, p. 67], hence we need to do some computations with it, but it is better suited for our purposes. It is the one considered for instance in [PaP1], to which we will refer frequently. The Siegel domain $\mathbb{H}_{\mathbb{C}}^{n}$ embeds in the complex projective $n$-space $\mathbb{P}_{n}(\mathbb{C})$ by the map (using homogeneous coordinates)

$$
\left(w_{0}, w\right) \mapsto\left[w_{0}: w: 1\right]
$$

We identify $\mathbb{H}_{\mathbb{C}}^{n}$ with its image by this map. This image, called the projective model of $\mathbb{H}_{\mathbb{C}}^{n}$ when endowed with the isometric Riemannian metric, is the negative cone of $q$, that is $\left\{\left[z_{0}: z: z_{n}\right] \in \mathbb{P}_{n}(\mathbb{C}): q\left(z_{0}, z, z_{n}\right)<0\right\}$. This embedding extends continuously to the boundary at infinity, by mapping $\left(w_{0}, w\right) \in \partial_{\infty} \mathbb{H}_{\mathbb{C}}^{n}-\{\infty\}$ to $\left[w_{0}: w: 1\right]$ and $\infty$ to $[1: 0: 0]$, so that the image of $\partial_{\infty} \mathbb{H}_{\mathbb{C}}^{n}$ is the null cone of $q$, that is $\left\{\left[z_{0}: z: z_{n}\right] \in \mathbb{P}_{n}(\mathbb{C})\right.$ : $\left.q\left(z_{0}, z, z_{n}\right)=0\right\}$.

The linear action of the special unitary group of $q$

$$
\mathrm{SU}_{q}=\left\{g \in \mathrm{SL}_{n+1}(\mathbb{C}): q \circ g=q\right\}
$$

on $\mathbb{C}^{n+1}$ induces a projective action on $\mathbb{P}_{n}(\mathbb{C})$. The quotient group $\mathrm{PSU}_{q}=\mathrm{SU}_{q} /\left(\mathbb{U}_{n+1} \mathrm{Id}\right)$ of $\mathrm{SU}_{q}$ by the kernel of the projective action, where $\mathbb{U}_{n+1}$ is the group of $(n+1)$-th roots of unity, preserves $\mathbb{H}_{\mathbb{C}}^{n}$, and its restriction to $\mathbb{H}_{\mathbb{C}}^{n}$ is the orientation-preserving isometry group of $\mathbb{H}_{\mathbb{C}}^{n}$. For instance by the paragraph above [PaP1, Lem. 6.3], an element $\gamma \in \mathrm{SU}_{q}$ fixes $\infty$ if and only if $\gamma$ is upper triangular (this is the reason, besides rationality problems, that we chose the Hermitian form $q$ rather than the one in [Gol]), see for instance [Gol, p. 119], [FaP, §2.1] up to signs.

By for instance [PaP1, Eq. (42)], the intersection of $\mathrm{SU}_{q}$ with the upper triangular subgroup of $\mathrm{SL}_{3}(\mathbb{C})$ is

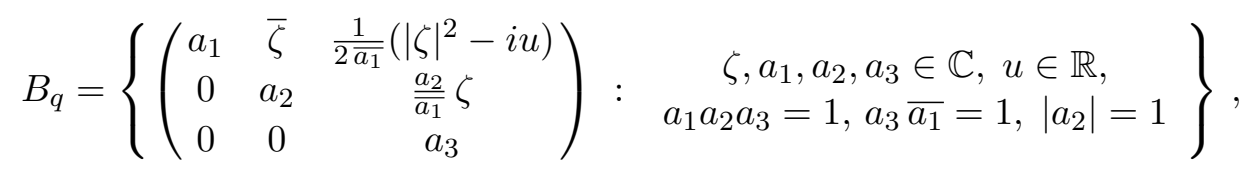

and its image $\overline{B_{q}}$ in $\mathrm{PSU}_{q}$ is equal to the stabiliser in $\mathrm{PSU}_{q}$ of $\infty$. 
If $\gamma \in \mathrm{SU}_{q}$ induces a loxodromic isometry on $\mathbb{H}_{\mathbb{C}}^{n}$, then $\gamma$ is diagonalisable over $\mathbb{C}$, it has a unique eigenvalue $\lambda$ of modulus $>1$, and its eigenvalues are $\lambda, \frac{1}{\lambda}, \frac{\bar{\lambda}}{\lambda}$ (see for instance [Par4, §3.2]). Furthermore, the translation length $\ell$ of $\gamma$ in $\mathbb{H}_{\mathbb{C}}^{n}$ is

$$
\ell=\ln |\lambda|
$$

(see for instance [Par4, Prop. 3.10], noting that this reference normalises the curvature to be between -1 and $-1 / 4)$.

The horospherical coordinates $(\zeta, u, t) \in \mathbb{C}^{n-1} \times \mathbb{R} \times[0,+\infty[$, that we will use from now on unless otherwise stated, of $\left(w_{0}, w\right) \in \mathbb{H}_{\mathbb{C}}^{n} \cup\left(\partial_{\infty} \mathbb{H}_{\mathbb{C}}^{n}-\{\infty\}\right)$ are

$$
(\zeta, u, t)=\left(w,-2 \operatorname{Im} w_{0}, 2 \operatorname{Re} w_{0}-|w|^{2}\right) \text { hence }\left(w_{0}, w\right)=\left(\frac{1}{2}\left(|\zeta|^{2}+t-i u\right), \zeta\right),
$$

so that the Riemannian metric is given by

$$
d s_{\mathbb{H}_{\mathbb{C}}^{n}}^{2}=\frac{1}{4 t^{2}}\left(d t^{2}+(d u+2 \operatorname{Im} d \zeta \cdot \bar{\zeta})^{2}+4 t d \zeta \cdot \overline{d \zeta}\right)
$$

In horospherical coordinates, the geodesic lines from $(\zeta, u, 0) \in \partial_{\infty} \mathbb{H}_{\mathbb{C}}^{n}-\{\infty\}$ to $\infty$ are, up to translations at the source, the map $s \mapsto\left(\zeta, u, e^{2 s}\right)$, by the normalisation of the metric. The closed horoballs centred at $\infty \in \partial_{\infty} \mathbb{H}_{\mathbb{C}}^{n}$ are the subsets

$$
\mathscr{H}_{s}=\left\{(\zeta, u, t) \in \mathbb{H}_{\mathbb{C}}^{n}: t \geq s\right\}
$$

and the horospheres centred at $\infty$ are their boundaries

$$
\partial \mathscr{H}_{s}=\left\{(\zeta, v, t) \in \mathbb{H}_{\mathbb{C}}^{n}: t=s\right\}
$$

for any $s>0$. Note that, for every $s \geq 1$, we have

$$
d\left(\partial \mathscr{H}_{1}, \partial \mathscr{H}_{s}\right)=\frac{\ln s}{2} .
$$

As introduced by [Par1, p. 297], the Cygan distance on $\mathbb{H}_{\mathbb{C}}^{n} \cup\left(\partial_{\infty} \mathbb{H}_{\mathbb{C}}^{n}-\{\infty\}\right.$ ) (analogous to the Euclidean distance on the closure in $\mathbb{R}^{n}$ of the upper halfspace model of $\left.\mathbb{H}_{\mathbb{R}}^{n}\right)$ is

$$
d_{\text {Cyg }}\left((\zeta, u, t),\left(\zeta^{\prime}, u^{\prime}, t^{\prime}\right)\right)=|| \zeta-\left.\zeta^{\prime}\right|^{2}+\left|t-t^{\prime}\right|+\left.i\left(u-u^{\prime}+2 \operatorname{Im} \zeta \cdot \overline{\zeta^{\prime}}\right)\right|^{1 / 2} .
$$

The Heisenberg group $\operatorname{Heis}_{2 n-1}$ of dimension $2 n-1$ is the real Lie group structure on $\mathbb{C}^{n-1} \times \mathbb{R}$ with law

$$
(\zeta, u)\left(\zeta^{\prime}, u^{\prime}\right)=\left(\zeta+\zeta^{\prime}, u+u^{\prime}+2 \operatorname{Im} \zeta \cdot \overline{\zeta^{\prime}}\right)
$$

and inverses $(\zeta, u)^{-1}=(-\zeta,-u)$. It identifies with $\partial_{\infty} \mathbb{H}_{\mathbb{C}}^{n}-\{\infty\}$ by the map $(\zeta, u) \mapsto$ $(\zeta, u, 0)$. It acts on $\mathbb{H}_{\mathbb{C}}^{n} \cup\left(\partial_{\infty} \mathbb{H}_{\mathbb{C}}^{n}-\{\infty\}\right)$ by the Heisenberg translations

$$
(\zeta, u)\left(\zeta^{\prime}, u^{\prime}, t^{\prime}\right)=\left(\zeta+\zeta^{\prime}, u+u^{\prime}+2 \operatorname{Im} \zeta \cdot \bar{\zeta}^{\prime}, t^{\prime}\right)
$$

that are isometries for both the Riemannian metric and the Cygan distance, and that preserve the horospheres centred at $\infty$. For every $u \in \mathbb{R}$, the Heisenberg translation by $(0, u)$ is called a vertical translation. 
It is easy to see that the Cygan distanc $2^{2}$ on $\operatorname{Heis}_{2 n-1}$ (see [Gol, page 160]) is the unique left-invariant distance on $\operatorname{Heis}_{2 n-1}$ with $d_{\mathrm{Cyg}}((\zeta, u),(0,0))=\left(|\zeta|^{4}+u^{2}\right)^{\frac{1}{4}}$. We introduced in [PaP1, §6.1] the modified Cygan distance $d_{\mathrm{Cyg}}^{\prime}$ as the unique left-invariant distance on Heis $_{2 n-1}$ with

$$
d_{\mathrm{Cyg}}^{\prime}((\zeta, u),(0,0))=\left(\left(|\zeta|^{4}+u^{2}\right)^{\frac{1}{2}}+|\zeta|^{2}\right)^{\frac{1}{2}} .
$$

We introduced in [PaP2, Lem. 3.4] the map $d_{\mathrm{Cyg}}^{\prime \prime}=\frac{d_{\mathrm{Cyg}}^{2}}{d_{\mathrm{Cyg}}^{\prime}}$, which is almost a distance on $\operatorname{Heis}_{2 n-1}$, as it satisfies $\frac{1}{\sqrt{2}} d_{\text {Cyg }} \leq d_{\text {Cyg }}^{\prime \prime} \leq d_{\text {Cyg }}$. For every nonempty subset $A$ of $\operatorname{Heis}_{2 n-1}$, we define the diameter of $A$ for this almost distance as

$$
\operatorname{diam}_{d_{\text {Cyg }}^{\prime \prime}}(A)=\sup _{x, y \in A} d_{\text {Cyg }}^{\prime \prime}(x, y) .
$$

The following result on diameters of finite chains will be useful in Section 7 .

Lemma 8 For every finite chain $C$, we have

$$
\operatorname{diam}_{d_{\text {Cyg }}}(C)=\frac{1}{\sqrt{2}} \operatorname{diam}_{d_{\text {Cyg }}^{\prime}}(C)=\sqrt{2} \operatorname{diam}_{d_{\text {Cyg }}^{\prime \prime}}(C) .
$$

Proof. For every chain $C$, there exists (see for instance [Gol, §3.1.4]) a unique point $P=\left[z_{0}: z: z_{n}\right]$ in $\mathbb{P}_{n}(\mathbb{C})$, called the polar point of $C$, such that $q\left(z_{0}: z: z_{n}\right)>0$ and $C$ is the intersection with $\partial_{\infty} \mathbb{H}_{\mathbb{C}}^{n}$ of the orthogonal to $P$ for $q$. Note that if $\gamma \in \mathrm{PSU}_{q}$, then the polar point of $\gamma C$ is $\gamma P$, and that $C$ is finite is and only if $z_{n} \neq 0$.

Let $P=\left[z_{0}: z: z_{n}\right]$ be the polar point of a finite chain $C$. Let $\gamma \in \mathrm{PSU}_{q}$ be the Heisenberg translation by

$$
\left[\frac{|z|^{2}}{2\left|z_{n}\right|^{2}}+i \operatorname{Im} \frac{z_{0}}{z_{n}}:-\frac{z}{z_{n}}: 1\right] \in \operatorname{Heis}_{2 n-1}
$$

An easy computation shows that $\gamma\left[z_{0}: z: z_{n}\right]=[-a: 0: b]$ with $a=q\left(z_{0}, z, z_{n}\right)>0$ and $b=2\left|z_{2}\right|^{2}>0$. Since the distances $d_{\mathrm{Cyg}}, d_{\mathrm{Cyg}}^{\prime}$ and $d_{\mathrm{Cyg}}^{\prime \prime}$ are left-invariant, we may assume that $P$ is the point $\left[-R^{2} / 2: 0: 1\right]$ with $R>0$. Hence, using the facts that Re $w_{0}=|w|^{2}$, $w=\zeta$ and $\operatorname{Im} w_{0}=-\frac{u}{2}$, we have

$$
C=\left\{\left[w_{0}: w: 1\right] \in \operatorname{Heis}_{2 n-1}: R^{2} / 2-\omega_{0}=0\right\}=\left\{(\zeta, u) \in \operatorname{Heis}_{2 n-1}: u=0,|\zeta|=R\right\},
$$

which is the sphere with radius $R$ in the first factor $\mathbb{C}^{n-1}$ of Heis $2 n-1$. Since the Heisenberg dilations $(\zeta, u) \mapsto\left(\lambda \zeta, \lambda^{2} u\right)$ with $\lambda>0$ are homotheties of ratio $\lambda$ for $d_{\text {Cyg }}, d_{\text {Cyg }}^{\prime}$ and $d_{\text {Cyg }}^{\prime \prime}$, we may assume that $R=1$. This proves in particular that the three diameters are proportional, and we now compute the proportionality constants.

For every $(\zeta, 0) \in C$, we have $d_{\text {Cyg }}((\zeta, 0),(0,0))=1$ and $d_{\text {Cyg }}^{\prime}((\zeta, 0),(0,0))=\sqrt{2}$, hence by the triangle inequality, $\operatorname{diam}_{d_{\mathrm{Cyg}}}(C) \leq 2$ and $\operatorname{diam}_{d_{\text {Cyg }}^{\prime}}(C) \leq 2 \sqrt{2}$. If $\zeta=(1,0, \ldots, 0)$, we have

$$
d_{\mathrm{Cyg}}((\zeta, 0),(-\zeta, 0))=d_{\mathrm{Cyg}}((2 \zeta, 0),(0,0))=2
$$

and similarly $d_{\text {Cyg }}^{\prime}((\zeta, 0),(-\zeta, 0))=2 \sqrt{2}$. Hence $\operatorname{diam}_{d_{\text {Cyg }}}(C)=2$ and $\operatorname{diam}_{d_{\text {Cyg }}^{\prime}}(C)=$ $2 \sqrt{2}$, which proves the first equality.

\footnotetext{
${ }^{2}$ It is called the Korányi distance by many people working in sub-Riemannian geometry, though Korányi Kor] does attribute it to Cygan Cyg.
} 
Assume first that $n \geq 3$. Since the group $U(n-1)$ acts transitively on the unit sphere of $\mathbb{C}^{n-1}$, since the stabiliser of $(1,0, \ldots, 0)$ in $U(n-1)$ acts transitively on the complex planes containing $(1,0, \ldots, 0)$ and on the real halflines of the second factor of $\mathbb{C}^{n-1}$, for all $\zeta, \zeta^{\prime} \in \mathbb{S}^{2 n-3}$, there exist $\gamma \in U(n-1), \theta \in[0,2 \pi]$ and $\varphi \in[0, \pi]$ such that

$$
\left(\gamma \zeta, \gamma \zeta^{\prime}\right)=\left((1,0, \ldots, 0),\left(e^{i \theta} \cos \varphi, \sin \varphi, 0, \ldots, 0\right)\right) .
$$

Hence we may assume that $n=3$, and with $\zeta_{\theta, \varphi}=\left(e^{i \theta} \cos \varphi, \sin \varphi\right)$, we have

$$
\operatorname{diam}_{d_{\text {Cyg }}^{\prime \prime}}(C)=\min _{\theta \in[0,2 \pi], \varphi \in[0, \pi]} d_{\text {Cyg }}^{\prime \prime}\left(\left(\zeta_{0,0}, 0\right),\left(\zeta_{\theta, \varphi}, 0\right)\right)
$$

Note that

$$
\left(-\zeta_{0,0}, 0\right) \cdot\left(\zeta_{\theta, \varphi}, 0\right)=\left(\zeta_{\theta, \varphi}-\zeta_{0,0},-2 \operatorname{Im} \zeta_{0,0} \cdot \overline{\zeta_{\theta, \varphi}}\right)=\left(\left(e^{i \theta} \cos \varphi-1, \sin \varphi\right), 2 \sin \theta \cos \varphi\right),
$$

and that $\left|\left(e^{i \theta} \cos \varphi-1, \sin \varphi\right)\right|^{2}=2(1-\cos \theta \cos \varphi)$. Therefore

$$
\begin{aligned}
d_{\text {Cyg }}^{\prime \prime}\left(\left(\zeta_{0,0}, 0\right),\left(\zeta_{\theta, \varphi}, 0\right)\right)^{2} & =d_{\text {Cyg }}^{\prime \prime}\left(\left(-\zeta_{0,0}, 0\right) \cdot\left(\zeta_{\theta, \varphi}, 0\right),(0,0)\right)^{2} \\
& =\frac{4(1-\cos \theta \cos \varphi)^{2}+4 \sin ^{2} \theta \cos ^{2} \varphi}{\left(4(1-\cos \theta \cos \varphi)^{2}+4 \sin ^{2} \theta \cos ^{2} \varphi\right)^{1 / 2}+2(1-\cos \theta \cos \varphi)} \\
& =\frac{2}{\frac{1}{\left(1+\cos ^{2} \varphi-2 \cos \theta \cos \varphi\right)^{1 / 2}}+\frac{1-\cos \theta \cos \varphi}{1+\cos ^{2} \varphi-2 \cos \theta \cos \varphi}} .
\end{aligned}
$$

Now $1+\cos ^{2} \varphi-2 \cos \theta \cos \varphi \leq 2(1-\cos \theta \cos \varphi) \leq 4$ and both equalities hold for instance if $\theta=\pi$ and $\varphi=0$. Hence $d_{\text {Cyg }}^{\prime \prime}\left(\left(\zeta_{0,0}, 0\right),\left(\zeta_{\theta, \varphi}, 0\right)\right)^{2} \leq 2$ with equality if $\theta=\pi$ and $\varphi=0$, therefore $\operatorname{diam}_{d_{\text {Cyg }}^{\prime \prime}}(C)=\sqrt{2}$. This proves the result when $n \geq 3$.

If $n=2$, for all $\zeta, \zeta^{\prime} \in \mathbb{S}^{1}$, there exist $\gamma \in U(1)$ and $\theta \in[0,2 \pi]$ such that $\left(\gamma \zeta, \gamma \zeta^{\prime}\right)=$ $\left(1, e^{i \theta}\right)$ and the result follows from the same computations as above with $\varphi=0$.

We conclude this section by two geometric lemmas that will be useful in Section 4. See also [Kim, §3], with slightly different conventions, for a computation similar to Lemma 9 based on [Gol, p. 113]. The Cygan distance, the Poisson kernel $e^{\beta_{(\xi, r)}}$, the Patterson measures $\mu_{x}$ computed in the next section, and related quantities are useful in potential theory on the Heisenberg group and for the study of the hypoelliptic Laplacian in subRiemannian geometry, see for instance [FS, Kra].

Lemma 9 For all $x=(\zeta, u, t)$ and $x^{\prime}=\left(\zeta^{\prime}, u^{\prime}, t^{\prime}\right)$ in $\mathbb{H}_{\mathbb{C}}^{n}$, for all $(\xi, r) \in \operatorname{Heis}_{2 n-1}=$ $\partial_{\infty} \mathbb{H}_{\mathbb{C}}^{n}-\{\infty\}$, we have

$$
\beta_{(\xi, r)}\left(x, x^{\prime}\right)=\frac{1}{2} \ln \frac{t^{\prime} d_{\mathrm{Cyg}}(x,(\xi, r))^{4}}{t d_{\mathrm{Cyg}}\left(x^{\prime},(\xi, r)\right)^{4}} .
$$

Proof. Since the Busemann function $\beta_{\infty}\left(x, x^{\prime}\right)$ is unchanged if $x, x^{\prime}$ are replaced by other points on the horospheres centred at $\infty$ through them, and since the map $s \mapsto\left(0,0, e^{2 s}\right)$ is a geodesic line in $\mathbb{H}_{\mathbb{C}}^{n}$ from $(0,0) \in \partial_{\infty} \mathbb{H}_{\mathbb{C}}^{n}$ to $\infty$, we have

$$
\beta_{\infty}\left(x, x^{\prime}\right)=\frac{1}{2} \ln \frac{t^{\prime}}{t}
$$


It is easy to check that the map $\iota:\left(w_{0}, w\right) \mapsto\left(\frac{1}{w_{0}}, \frac{w}{w_{0}}\right)$ is an isometric involution of $\mathbb{H}_{\mathbb{C}}^{n}$ sending $(0,0) \in \partial_{\infty} \mathbb{H}_{\mathbb{C}}^{n}$ to $\infty$. Hence, with $x=\left(w_{0}, w\right)$ and $x^{\prime}=\left(w_{0}^{\prime}, w^{\prime}\right)$, using Equation (7) and the fact that $d_{\mathrm{Cyg}}(x,(0,0))^{4}=4\left|w_{0}\right|^{2}$ and $d_{\mathrm{Cyg}}\left(x^{\prime},(0,0)\right)^{4}=4\left|w_{0}^{\prime}\right|^{2}$, we have

$$
\beta_{(0,0)}\left(x, x^{\prime}\right)=\beta_{\iota(0,0)}\left(\iota x, \iota x^{\prime}\right)=\frac{1}{2} \ln \frac{2 \operatorname{Re} \frac{1}{w_{0}^{\prime}}-\left|\frac{w^{\prime}}{w_{0}^{\prime}}\right|^{2}}{2 \operatorname{Re} \frac{1}{w_{0}}-\left|\frac{w}{w_{0}}\right|^{2}}=\frac{1}{2} \ln \frac{t^{\prime} d_{\mathrm{Cyg}}(x,(0,0))^{4}}{t d_{\mathrm{Cyg}}\left(x^{\prime},(0,0)\right)^{4}} .
$$

The Heisenberg translation $\tau$ by $(\xi, r)$ preserves the last horospherical coordinates and the Cygan distances. Thus, $\beta_{(\xi, r)}\left(x, x^{\prime}\right)=\beta_{(0,0)}\left(\tau^{-1} x, \tau^{-1} x^{\prime}\right)$, which implies the claim.

Lemma 10 The orthogonal projection from $\partial_{\infty} \mathbb{H}_{\mathbb{C}}^{n}-\{(0,0), \infty\}$ to the geodesic line in $\mathbb{H}_{\mathbb{C}}^{n}$ with points at infinity $(0,0)$ and $\infty$ is $\left(w_{0}, w\right) \mapsto\left(2\left|w_{0}\right|, 0\right)$, that is, in horospherical coordinates, $(\zeta, u, 0) \mapsto\left(0,0,\left(|\zeta|^{4}+u^{2}\right)^{1 / 2}\right)$.

In particular, the preimages by this orthogonal projection are the spheres of center $(0,0)$ for the Cygan distance on $\mathrm{Heis}_{2 n-1}$. They are spinal spheres with complex spine $\left\{\left(w_{0}, w\right) \in \mathbb{H}_{\mathbb{C}}^{n}: w=0\right\}$, see [Gol, $\left.\S 5.1 .9\right]$.

Proof. For every parameter $a$ ranging in $] 0,+\infty\left[\right.$, consider the horosphere $\partial \mathscr{H}_{a}$ centred at $\infty$. Its image by the isometric involution $\iota:\left(w, w_{0}\right) \mapsto\left(\frac{1}{w_{0}}, \frac{w}{w_{0}}\right)$ is, using Equation (7), the horosphere $\left\{(\xi, r, t) \in \mathbb{H}_{\mathbb{C}}^{n}: t=\frac{a}{4}\left(\left(|\xi|^{2}+t\right)^{2}+r^{2}\right)\right\}$ centred at $(0,0)$. The image of this horosphere by the Heisenberg translation by $(\zeta, u)$ is the horosphere

$$
\left\{(\xi, r, t) \in \mathbb{H}_{\mathbb{C}}^{n}: t=\frac{a}{4}\left(\left(|-\zeta+\xi|^{2}+t\right)^{2}+(-u+r-2 \operatorname{Im} \zeta \cdot \bar{\xi})^{2}\right)\right\}
$$

centred at $(\zeta, u)$. The orthogonal projection of $(\zeta, u)$ on the geodesic line $\ell$ from $(0,0)$ to $\infty$ is attained when the parameter $a$ gives a double point of intersection $(0,0, t)$ between this horosphere and $\ell$. The quadratic equation

$$
t=\frac{a}{4}\left(\left(|\zeta|^{2}+t\right)^{2}+u^{2}\right)
$$

with unknown $t$ has a double solution if and only if its reduced discriminant $\Delta^{\prime}=\left(|\zeta|^{2}-\frac{2}{a}\right)^{2}-\left(|\zeta|^{4}+u^{2}\right)$ vanishes, that is, since $a>0$, if and only if $a=\frac{2}{\left(|\zeta|^{4}+u^{2}\right)^{1 / 2}+|\zeta|^{2}}$, giving $(0,0, t)$

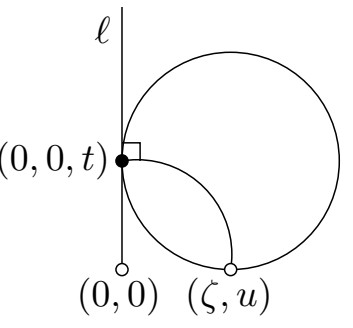
$t=\left(|\zeta|^{4}+u^{2}\right)^{1 / 2}$. The result follows.

\section{Measure computations in complex hyperbolic spaces}

In this Section, we give proportionality constants relating, on the one hand, Patterson, Bowen-Margulis and skinning measures associated to some convex subsets and, on the other hand, the corresponding Riemannian measures, in the complex hyperbolic case.

We will denote the standard Lebesgue measure on $\mathbb{C}^{n-1}$ by $d \zeta$, and the usual left Haar measure on $\operatorname{Heis}_{2 n-1}$ by

$$
d \lambda_{2 n-1}(\zeta, u)=d \zeta d u .
$$

In horospherical coordinates, the volume form of $\left.\mathbb{H}_{\mathbb{C}}^{n}=\operatorname{Heis}_{2 n-1} \times\right] 0,+\infty[$ is

$$
d \operatorname{vol}_{\mathbb{H}_{\mathbb{C}}^{n}}(\zeta, u, t)=\frac{1}{4 t^{n+1}} d \zeta d u d t
$$


We begin by recalling a lemma that relates the volume of a Margulis cusp neighbourhood with the volume of its boundary (compare [HeP2, Lem. 5.2]).

Lemma 11 Let $D$ be a horoball in $\mathbb{H}_{\mathbb{C}}^{n}$ and let $\Gamma$ be a discrete group of isometries of $\mathbb{H}_{\mathbb{C}}^{n}$ preserving $\partial D$ (hence $D)$. Then $\operatorname{Vol}(\Gamma \backslash \partial D)=2 n \operatorname{Vol}(\Gamma \backslash D)$.

Proof. Since the group of isometries of $\mathbb{H}_{\mathbb{C}}^{n}$ acts transitively on the set of horospheres of $\mathbb{H}_{\mathbb{C}}^{n}$, we may assume that $D=\mathscr{H}_{1}$. The horosphere centred at $\infty$ through a point $(\zeta, u, t) \in \mathscr{H}_{1}$ is $\partial \mathscr{H}_{t}$ and its orthogonal geodesic line at this point is $s \mapsto\left(\zeta, u, e^{2 s}\right)$, hence

$$
d \operatorname{vol}_{\mathbb{H}_{\mathbb{C}}^{n}}(\zeta, u, t)=d \operatorname{vol}_{\partial \mathscr{H}_{t}}(\zeta, u, t) \frac{d t}{2 t}
$$

By Equation (10), we hence have

$$
d \operatorname{vol}_{\partial \mathscr{H}_{t}}(\zeta, u, t)=\frac{1}{2 t^{n}} d \zeta d u
$$

for every $t>0$, therefore $d \operatorname{vol}_{\partial \mathscr{H}_{t}}(\zeta, u, t)=\frac{1}{t^{n}} d \operatorname{vol}_{\partial \mathscr{H}_{1}}(\zeta, u, 1)$. The homeomorphism from $\partial \mathscr{H}_{t}$ to $\partial \mathscr{H}_{1}$ defined by $(\zeta, u, t) \mapsto(\zeta, u, 1)$ commutes with the action of $\Gamma$. Thus,

$$
\begin{aligned}
\operatorname{Vol}\left(\Gamma \backslash \mathscr{H}_{1}\right) & =\int_{\Gamma \backslash \mathscr{H}_{1}} d \operatorname{vol}_{\mathbb{H}_{\mathbb{C}}^{n}}(\zeta, u, t)=\int_{t=1}^{+\infty} \int_{\Gamma \backslash \partial \mathscr{H}_{t}} d \operatorname{vol}_{\partial \mathscr{H}_{t}}(\zeta, u, t) \frac{d t}{2 t} \\
& =\int_{t=1}^{+\infty} \int_{\Gamma \backslash \partial \mathscr{H}_{1}} d \operatorname{vol}_{\partial \mathscr{H}_{1}}(\zeta, u, 1) \frac{d t}{2 t^{n+1}}=\frac{1}{2 n} \operatorname{Vol}\left(\Gamma \backslash \partial \mathscr{H}_{1}\right)
\end{aligned}
$$

Let $\Gamma$ be a lattice in Isom $\left(\mathbb{H}_{\mathbb{C}}^{n}\right)$. Its critical exponent is $\delta_{\Gamma}=2 n$ (see for instance [CI, §6]). The Patterson density $\left(\mu_{x}\right)_{x \in \mathbb{H}_{\mathbb{C}}^{n}}$ of $\Gamma$ is uniquely defined up to a multiplicative constant, and is independent of $\Gamma$. We will choose the normalisation such that the measure $\mu_{\infty}$ defined in Remark 6 by the horoball $\mathscr{H}_{1}$ coincides with $\lambda_{2 n-1}$ on $\partial_{\infty} \mathbb{H}_{\mathbb{C}}^{n}-\{\infty\}=\operatorname{Heis}_{2 n-1}$, that is

$$
d \mu_{\infty}(\xi, r)=d \lambda_{2 n-1}(\xi, r)=d \xi d r .
$$

This is possible since $\mu_{\infty}$ is invariant under the isometry group Heis Hen-1 $_{2 n}$ preserving $\mathscr{H}_{1}$, hence it is a Haar measure on $\partial_{\infty} \mathbb{H}_{\mathbb{C}}^{n}-\{\infty\}=$ Heis $_{2 n-1}$.

Lemma 12 Let $\Gamma$ be a lattice in $\operatorname{Isom}\left(\mathbb{H}_{\mathbb{C}}^{n}\right)$, and let $\mu_{\infty}$ be normalised as above. For all $x=(\zeta, u, t)$ and $x^{\prime}=\left(\zeta^{\prime}, u^{\prime}, t^{\prime}\right)$ in $\mathbb{H}_{\mathbb{C}}^{n}$, for all $(\xi, r) \in \partial_{\infty} \mathbb{H}_{\mathbb{C}}^{n}-\{\infty\}$ and $v \in T^{1} \mathbb{H}_{\mathbb{C}}^{n}$ such that $v_{ \pm} \neq \infty$, we have

(i) $d \mu_{x}(\xi, r)=\frac{t^{n}}{d_{\mathrm{Cyg}}(x,(\xi, r))^{4 n}} d \xi d r$;

(ii) using a Hopf parametrisation $v \mapsto\left(v_{-}, v_{+}, s\right)$,

$$
d \widetilde{m}_{\mathrm{BM}}(v)=\frac{d \lambda_{2 n-1}\left(v_{-}\right) d \lambda_{2 n-1}\left(v_{+}\right) d s}{d_{\mathrm{Cyg}}\left(v_{-}, v_{+}\right)^{4 n}}
$$

(iii)

$$
\widetilde{m}_{\mathrm{BM}}=\frac{2 n-1}{2^{2 n-2}} \operatorname{vol}_{T^{1} \mathbb{H}_{\mathbb{C}}^{n}},
$$


and in particular

$$
\left\|m_{\mathrm{BM}}\right\|=\frac{(2 n-1) \pi^{n}}{2^{2 n-3}(n-1) !} \operatorname{Vol}(M)
$$

(iv) using the homeomorphism $v \mapsto v_{+}$from $\partial_{+}^{1} \mathscr{H}_{1}$ to $\partial_{\infty} \mathbb{H}_{\mathbb{C}}^{n}-\{\infty\}=\operatorname{Heis}_{2 n-1}$,

$$
d \widetilde{\sigma}_{\mathscr{H}_{1}}(v)=d \lambda_{2 n+1}\left(v_{+}\right),
$$

and for every horoball $D^{-}$in $\mathbb{H}_{\mathbb{C}}^{n}$,

$$
\pi_{*} \widetilde{\sigma}_{D^{-}}=2 \operatorname{vol}_{\partial D^{-}},
$$

and

$$
\left\|\sigma_{D^{-}}\right\|=4 n \operatorname{Vol}\left(\Gamma_{D^{-}} \backslash D^{-}\right)
$$

(v) for every geodesic line $D^{-}$in $\mathbb{H}_{\mathbb{C}}^{n}$,

$$
d \pi_{*} \widetilde{\sigma}_{D^{-}}=\frac{n}{4^{n-1}(2 n-1)} d \pi_{*} \operatorname{vol}_{\partial_{+}^{1} D^{-}}
$$

and, with $m$ the order of the pointwise stabiliser of $D^{-}$in $\Gamma$,

$$
\left\|\sigma_{D^{-}}\right\|=\frac{2 \pi^{n-1} n !}{m(2 n-1) !} \operatorname{Vol}\left(\Gamma_{D^{-}} \backslash D^{-}\right)
$$

(vi) for every complex geodesic line $D^{-}$in $\mathbb{H}_{\mathbb{C}}^{n}$, we have

$$
d \pi_{*} \widetilde{\sigma}_{D^{-}}=\frac{1}{2^{2 n-3}} d \pi_{*} \operatorname{vol}_{\partial_{+}^{1} D^{-}}
$$

and, with $m$ the order of the pointwise stabiliser of $D^{-}$in $\Gamma$,

$$
\left\|\sigma_{D^{-}}\right\|=\frac{\pi^{n-1}}{m 4^{n-2}(n-2) !} \operatorname{Vol}\left(\Gamma_{D^{-}} \backslash D^{-}\right) .
$$

Proof. In the computations below, it is useful to note that Lemma 9 implies that

$$
e^{-2 n \beta_{(\xi, r)}\left(x, x^{\prime}\right)}=\frac{t^{n} d_{\mathrm{Cyg}}\left(x^{\prime},(\xi, r)\right)^{4 n}}{\left(t^{\prime}\right)^{n} d_{\mathrm{Cyg}}(x,(\xi, r))^{4 n}} .
$$

(i) The geodesic line from $(\xi, r)$ to $\infty$ goes through $\partial \mathscr{H}_{1}$ at the point $(\xi, r, 1)$. By the normalisation of $d \mu_{\infty}$ and by Equation (3), we hence have

$$
\frac{d \mu_{x}}{d \xi d r}(\xi, r)=\frac{d \mu_{x}}{d \mu_{\infty}}(\xi, r)=e^{-2 n \beta_{(\xi, r)}(x,(\xi, r, 1))} .
$$

The result then follows from Equation (12). 
(ii) Note that if $x^{\prime}$ is on the geodesic line defined by $v$, then $d_{\text {Cyg }}\left(x^{\prime}, v_{-}\right)^{2} \sim t^{\prime}$ as $x^{\prime} \rightarrow v_{-}$. Hence, by Equation (12) and Assertion (i), by letting $x^{\prime}$ converge to $v_{-}$on the geodesic line defined by $v$, we have

$$
\begin{aligned}
& d \widetilde{m}_{\mathrm{BM}}(v)=e^{-2 n\left(\beta_{v_{-}}\left(x^{\prime}, x\right)+\beta_{v_{+}}\left(x^{\prime}, x\right)\right)} d \mu_{x}\left(v_{-}\right) d \mu_{x}\left(v_{+}\right) d s \\
& =\left(\frac{t^{\prime} d_{\mathrm{Cyg}}\left(x, v_{-}\right)^{4} t^{\prime} d_{\mathrm{Cyg}}\left(x, v_{+}\right)^{4} t^{2}}{t d_{\mathrm{Cyg}}\left(x^{\prime}, v_{-}\right)^{4} t d_{\mathrm{Cyg}}\left(x^{\prime}, v_{+}\right)^{4} d_{\mathrm{Cyg}}\left(x, v_{-}\right)^{4} d_{\mathrm{Cyg}}\left(x, v_{+}\right)^{4}}\right)^{n} d \lambda_{2 n-1}\left(v_{-}\right) d \lambda_{2 n-1}\left(v_{+}\right) d s \\
& =\frac{1}{d_{\mathrm{Cyg}}\left(v_{-}, v_{+}\right)^{4 n}} d \lambda_{2 n-1}\left(v_{-}\right) d \lambda_{2 n-1}\left(v_{+}\right) d s .
\end{aligned}
$$

(iii) Recall that the Liouville measure $\operatorname{vol}_{T^{1} \mathbb{H}_{\mathbb{C}}^{n}}$ (which is the Riemannian measure for Sasaki's metric on $T^{1} \mathbb{H}_{\mathbb{C}}^{n}$ ) disintegrates under the fibration $\pi: T^{1} \mathbb{H}_{\mathbb{C}}^{n} \rightarrow \mathbb{H}_{\mathbb{C}}^{n}$ over the Riemannian measure $\operatorname{vol}_{\mathbb{H}_{\mathbb{C}}^{n}}$ of $\mathbb{H}_{\mathbb{C}}^{n}$, with conditional measures the spherical measures on the unit tangent spheres:

$$
d \operatorname{vol}_{T^{1} \mathbb{H}_{\mathbb{C}}^{n}}(v)=\int_{x \in \mathbb{H}_{\mathbb{C}}^{n}} d \operatorname{vol}_{T_{x}^{1} \mathbb{H}_{\mathbb{C}}^{n}}(v) d \operatorname{vol}_{\mathbb{H}_{\mathbb{C}}^{n}}(x) .
$$

Let $x \in \mathbb{H}_{\mathbb{C}}^{n}$ with last horospherical coordinate $t$. Since the group $I_{x}$ of isometries of $\mathbb{H}_{\mathbb{C}}^{n}$ fixing $x$ acts transitively on $T_{x}^{1} \mathbb{H}_{\mathbb{C}}^{n}$, and both $\mu_{x}$ and the Riemannian measure $\operatorname{vol}_{T_{x}^{1} \mathbb{H}_{\mathbb{C}}^{n}}$ are invariant under $I_{x}$, using the $I_{x}$-equivariant homeomorphism $v \mapsto v_{+}$from $T_{x}^{1} \mathbb{H}_{\mathbb{C}}^{n}$ to $\partial_{\infty} \mathbb{H}_{\mathbb{C}}^{n}$, we have, for all $v \in T_{x}^{1} \mathbb{H}_{\mathbb{C}}^{n}$ such that $v_{+} \neq \infty$, using Assertion (i) for the last equality,

$$
d \operatorname{vol}_{T_{x}^{1} \mathbb{H}_{\mathbb{C}}^{n}}(v)=\frac{\operatorname{Vol}\left(\mathbb{S}^{2 n-1}\right)}{\left\|\mu_{x}\right\|} d \mu_{x}\left(v_{+}\right)=\frac{\operatorname{Vol}\left(\mathbb{S}^{2 n-1}\right) t^{n}}{\left\|\mu_{x}\right\| d_{\mathrm{Cyg}}\left(x, v_{+}\right)^{4 n}} d \lambda_{2 n-1}\left(v_{+}\right) .
$$

By homogeneity and by Assertion (i), we have,

$$
\begin{aligned}
\left\|\mu_{x}\right\| & =\left\|\mu_{(0,0,1)}\right\|=\int_{\mathbb{C}^{n-1} \times \mathbb{R}} \frac{d \xi d r}{\left(\left(|\xi|^{2}+1\right)^{2}+r^{2}\right)^{n}} \\
& =2 \operatorname{Vol}\left(\mathbb{S}^{2 n-3}\right) \int_{\left[0,+\infty\left[^{2}\right.\right.} \frac{\rho^{2 n-3} d \rho d r}{\left(\left(\rho^{2}+1\right)^{2}+r^{2}\right)^{n}} .
\end{aligned}
$$

Using Mathematica for the first equation and the well known expression of $\Gamma(n+1 / 2)$ for the second one, we have

$$
\begin{aligned}
2 \int_{\left[0,+\infty\left[^{2}\right.\right.} \frac{s^{n-2} d s d r}{\left(s^{2}+r^{2}+2 s+1\right)^{n}} & =\frac{\sqrt{\pi}(n-2) ! \Gamma(n+1 / 2)}{(2 n-2) !} \\
& =\frac{\sqrt{\pi}(n-2) !}{(2 n-2) !} \frac{(2 n) ! \sqrt{\pi}}{2^{2 n} n !}=\frac{(2 n-1) \pi}{2^{2 n-1}(n-1)} .
\end{aligned}
$$

Using the change of variable $s=\rho^{2}$, since $\operatorname{Vol}\left(\mathbb{S}^{2 n-1}\right)=\frac{\pi}{n-1} \operatorname{Vol}\left(\mathbb{S}^{2 n-3}\right)$, we hence have

$$
\left\|\mu_{x}\right\|=\frac{(2 n-1)}{2^{2 n-1}} \operatorname{Vol}\left(\mathbb{S}^{2 n-1}\right) \text {. }
$$

Hence, by Equations (10) and (13), using the homeomorphism $v \mapsto\left(v_{+}, \pi(v)=(\zeta, u, t)\right)$ from $T^{1} \mathbb{H}_{\mathbb{C}}^{n}$ to $\partial_{\infty} \mathbb{H}_{\mathbb{C}}^{n} \times \mathbb{H}_{\mathbb{C}}^{n}$, we have, for all $v \in T^{1} \mathbb{H}_{\mathbb{C}}^{n}$ such that $v_{+} \neq \infty$

$$
d \operatorname{vol}_{T^{1} \mathbb{H}_{\mathbb{C}}^{n}}(v)=\frac{2^{2 n-3}}{(2 n-1) t d_{\mathrm{Cyg}}\left((\zeta, u, t), v_{+}\right)^{4 n}} d \lambda_{2 n-1}\left(v_{+}\right) d \zeta d u d t
$$


Consider the map $F: \operatorname{Heis}_{2 n-1} \times \mathbb{R} \rightarrow \mathbb{H}_{\mathbb{C}}^{n}$ defined by

$$
(\xi, r, s) \mapsto\left(\zeta=\frac{\xi}{1+\left(|\xi|^{2}-i r\right) e^{2 s}}, u=-\operatorname{Im} \frac{|\xi|^{2}-i r}{1+\left(|\xi|^{2}-i r\right) e^{2 s}}, t=\frac{e^{2 s}\left(|\xi|^{4}+r^{2}\right)}{\left|1+\left(|\xi|^{2}-i r\right) e^{2 s}\right|^{2}}\right) .
$$

Note that $F(0,1,0)=\left(0, \frac{1}{2}, \frac{1}{2}\right)$. By the first two centred formulas of [PaP2, page 113], applied with the term $t$ in these formulas equal to $2 s+\ln 2$ and using Equation (7), noting that the sectional curvature in $[\mathrm{PaP} 2]$ is normalised to be in $\left[-1,-\frac{1}{4}\right]$, the map $s \mapsto F(\xi, r, s)$ is a geodesic line starting from $(\xi, r)$ and ending in $(0,0)$. On this geodesic, $s$ and the time parameter in Hopf's parametrisation differ only by an additive constant, hence have the same differential.

Recall that by homogeneity, the two measures $\widetilde{m}_{\mathrm{BM}}$ and $\operatorname{vol}_{T^{1} \mathbb{H}_{\mathbb{C}}^{n}}$ are proportional. Hence, computing their (constant) Radon-Nikodym derivative at $v \in T^{1} \mathbb{H}_{\mathbb{C}}^{n}$ such that $v_{-}=(0,1)$ and $\pi(v)=\left(0, \frac{1}{2}, \frac{1}{2}\right)$ (so that $v$ is tangent to the geodesic line $s \mapsto F(0,1, s)$ at $s=0$, hence $\left.v_{+}=(0,0)\right)$, we have, by Assertion (ii) with $(\xi, r)$ parametrizing $v_{-}$and by Equation (14),

$$
\begin{aligned}
\frac{d \operatorname{vol}_{T^{1} \mathbb{H}_{\mathbb{C}}^{n}}}{d \widetilde{m}_{\mathrm{BM}}} & =\frac{2^{2 n-3} d_{\mathrm{Cyg}}((0,1,0),(0,0,0))^{4 n}}{(2 n-1) \frac{1}{2} d_{\mathrm{Cyg}}\left(\left(0, \frac{1}{2}, \frac{1}{2}\right),(0,0,0)\right)^{4 n}} \frac{d \zeta d u d t}{d \xi d r d s}(0,1,0) \\
& =\frac{2^{3 n-2}}{(2 n-1)} \frac{d \zeta d u d t}{d \xi d r d s}(0,1,0) .
\end{aligned}
$$

The first claim of Assertion (iii) follows by computing the Jacobian at $(0,1,0)$ of the map $F$, which is equal to $\frac{1}{2^{n}}$, since at the point $(0,1,0)$, we have

$$
\frac{\partial \zeta}{\partial \xi}=\frac{1}{1-i} \operatorname{Id}_{\mathbb{C}^{n-1}}, \quad \frac{\partial \zeta}{\partial r}=\frac{\partial \zeta}{\partial s}=0, \quad \frac{\partial u}{\partial r}=0, \quad \frac{\partial u}{\partial s}=-1, \quad \frac{\partial t}{\partial r}=\frac{1}{2} .
$$

The second claim follows from the facts that $\operatorname{Vol}\left(T^{1} M\right)=\operatorname{Vol}\left(\mathbb{S}^{2 n-1}\right) \operatorname{Vol}(M)$ and that $\operatorname{Vol}\left(\mathbb{S}^{2 n-1}\right)=\frac{2 \pi^{n}}{(n-1) !}$.

(iv) The first claim follows from Equation (5). By Equation (11), we have

$$
d \operatorname{vol}_{\partial \mathscr{H}_{1}}(\zeta, u, 1)=\frac{1}{2} d \zeta d u .
$$

Hence $\pi_{*} \widetilde{\sigma}_{\mathscr{H}_{1}}=2 \operatorname{vol}_{\partial \mathscr{H}_{1}}$, and by the transitivity of the isometry group of $\mathbb{H}_{\mathbb{C}}^{n}$ on the set of horoballs in $\mathbb{H}_{\mathbb{C}}^{n}$, the second claim of Assertion (iv) follows. Therefore, by Lemma 11 .

$$
\left\|\sigma_{D^{-}}\right\|=\left\|\pi_{*} \sigma_{D^{-}}\right\|=2 \operatorname{Vol}\left(\Gamma_{D^{-}} \backslash \partial D^{-}\right)=4 n \operatorname{Vol}\left(\Gamma_{D^{-}} \backslash D^{-}\right) .
$$

(v) By the transitivity of the isometry group of $\mathbb{H}_{\mathbb{C}}^{n}$ on the set of its geodesic lines, we may assume that $D^{-}$is the geodesic line in $\mathbb{H}_{\mathbb{C}}^{n}$ with points at infinity $(0,0)$ and $\infty$. The map from the full-measure open subset $\left\{(\zeta, u) \in \operatorname{Heis}_{2 n-1}: \zeta \neq 0\right\}$ in Heis $2 n-1$ to the product manifold $\left.\mathbb{S}^{2 n-3} \times\right] 0,+\infty[\times]-\frac{\pi}{2}, \frac{\pi}{2}[$ defined by

$$
(\zeta, u) \mapsto\left(\sigma=\frac{\zeta}{|\zeta|}, \rho=\left(|\zeta|^{4}+u^{2}\right)^{1 / 2}, \theta=\arctan \frac{u}{|\zeta|^{2}}\right)
$$


is a diffeomorphism. Since $|\zeta|=\sqrt{\rho \cos \theta}$ and $u=\rho \sin \theta$, we have

$$
d \zeta d u=\frac{1}{2}|\zeta|^{2 n-4} d \operatorname{vol}_{\mathbb{S}^{2 n-3}}\left(\frac{\zeta}{|\zeta|}\right) d\left(|\zeta|^{2}\right) d u=\frac{1}{2}(\rho \cos \theta)^{n-2} \rho d \operatorname{vol}_{\mathbb{S}^{2 n-3}}(\sigma) d \rho d \theta .
$$

By Lemma 10, by Equation (12) and by Assertion (i), using the homeomorphism sending $v \in \partial_{+}^{1} D^{-}$to $v_{+}=(\zeta, u) \in \operatorname{Heis}_{2 n-1}-\{(0,0)\}$ and the inverse transformation $|\zeta|^{2}=\rho \cos \theta$, $u=\rho \sin \theta$, we have

$$
\begin{aligned}
d \widetilde{\sigma}_{D^{-}}(v) & =e^{-2 n \beta_{(\zeta, u)}\left(\left(0,0,\left(|\zeta|^{4}+u^{2}\right)^{1 / 2}\right),(0,0,1)\right)} d \mu_{(0,0,1)}(\zeta, u) \\
& =\frac{\left(|\zeta|^{4}+u^{2}\right)^{n / 2} d \zeta d u}{d_{\mathrm{Cyg}}\left(\left(0,0,\left(|\zeta|^{4}+u^{2}\right)^{1 / 2}\right),(\zeta, u, 0)\right)^{4 n}}=\left(\frac{\left(|\zeta|^{4}+u^{2}\right)^{1 / 2}}{\left(|\zeta|^{2}+\left(|\zeta|^{4}+u^{2}\right)^{1 / 2}\right)^{2}+u^{2}}\right)^{n} d \zeta d u \\
& =\frac{\cos ^{n-2} \theta}{2^{n+1}(1+\cos \theta)^{n}} d \operatorname{vol}_{\mathbb{S}^{2 n-3}}(\sigma) \frac{d \rho}{\rho} d \theta .
\end{aligned}
$$

Thus,

$$
d \pi_{*} \widetilde{\sigma}_{D^{-}}(0,0, \rho)=\frac{c_{n}^{\prime} \operatorname{Vol}\left(\mathbb{S}^{2 n-3}\right)}{2^{n+1}} \frac{d \rho}{\rho}
$$

where, using Mathematica,

$$
c_{n}^{\prime}=\int_{-\frac{\pi}{2}}^{\frac{\pi}{2}} \frac{\cos ^{n-2} \theta}{(1+\cos \theta)^{n}} d \theta=\frac{2^{1-n} \sqrt{\pi} n !}{(n-1) \Gamma\left(n+\frac{1}{2}\right)} .
$$

The next step is to obtain a similar expression for the Riemannian measure of the submanifold $\partial_{+}^{1} D^{-}$of $T^{1} \mathbb{H}_{\mathbb{C}}^{n}$ (endowed with Sasaki's metric). For every $x \in D^{-}$, let us denote by $\nu_{x}^{1} D^{-}$the fiber over $x$ of the normal bundle map $v \mapsto \pi(v)$ from $\partial_{+}^{1} D^{-}$to $D^{-}$. We endow $\nu_{x}^{1} D^{-}$with the spherical metric induced by the scalar product of the tangent space $T_{x} \mathbb{H}_{\mathbb{C}}^{n}$ at $x$. The Riemannian measure of $\partial_{+}^{1} D^{-}$disintegrates under this fibration over the Riemannian measure of $D^{-}$as

$$
d \operatorname{vol}_{\partial_{+}^{1} D^{-}}(v)=\int_{x \in D^{-}} d \operatorname{vol}_{\nu_{x}^{1} D^{-}}(v) d \operatorname{vol}_{D^{-}}(x) .
$$

By looking at the expression (8) of the Riemannian metric of $\mathbb{H}_{\mathbb{C}}^{n}$ in horospherical coordinates, using the homeomorphism $\rho \mapsto x=(0,0, \rho)$ from $\left[0,+\infty\left[\right.\right.$ to $D^{-}$, we have

$$
d \operatorname{vol}_{D^{-}}(x)=\frac{d \rho}{2 \rho} .
$$

Hence

$$
d \pi_{*} \operatorname{vol}_{\partial_{+}^{1} D^{-}}(0,0, \rho)=\operatorname{Vol}\left(\mathbb{S}^{2 n-2}\right) \frac{d \rho}{2 \rho} .
$$

We have $\operatorname{Vol}\left(\mathbb{S}^{2 n-2}\right)=\frac{2 \pi^{n-\frac{1}{2}}}{\Gamma\left(n-\frac{1}{2}\right)}=\frac{2^{2 n-1} \pi^{n-1}(n-1) !}{(2 n-2) !}$ and $\operatorname{Vol}\left(\mathbb{S}^{2 n-3}\right)=\frac{2 \pi^{n-1}}{(n-2) !}$. Since $\Gamma(x+1)=$ $x \Gamma(x)$ for all $x>0$, Equations (17) and (18) give the first claim of Assertion (v).

The second one follows, since pushforwards of measures preserves their total mass, and since $\operatorname{Vol}\left(\Gamma_{\partial_{+}^{1} D^{-}} \backslash \partial_{+}^{1} D^{-}\right)=\frac{\operatorname{Vol}\left(\mathbb{S}^{2 n-2}\right)}{m} \operatorname{Vol}\left(\Gamma_{D^{-}} \backslash D^{-}\right)$.

(vii) By the transitivity of the isometry group of $\mathbb{H}_{\mathbb{C}}^{n}$ on the set of its complex geodesic lines, we may assume that $D^{-}$is the complex geodesic line $C=\left\{\left(w_{0}, w\right) \in \mathbb{H}_{\mathbb{C}}^{n}: w=0\right\}$ 
or, in horospherical coordinates, $C=\left\{(\zeta, u, t) \in \mathbb{H}_{\mathbb{C}}^{n}: \zeta=0\right\}$. By [Gol, p. 157], the orthogonal projection of $\mathbb{H}_{\mathbb{C}}^{n}$ to $C$ is the map $\left(w_{0}, w\right) \mapsto\left(w_{0}, 0\right)$, which in horospherical coordinates on $\partial_{\infty} \mathbb{H}_{\mathbb{C}}^{n}-\partial_{\infty} C$ extends as $(\zeta, u, 0) \mapsto\left(0, u,|\zeta|^{2}\right)$.

Hence, using the homeomorphism from $\partial_{+}^{1} C$ to $\left\{(\zeta, u) \in\right.$ Heis $\left._{2 n-1}: \zeta \neq 0\right\}$ sending a normal unit vector $v$ to its point at infinity $v_{+}=(\zeta, u)$, we have, by Equation 12 and by Assertion (i), 9 ,

$$
\begin{aligned}
d \widetilde{\sigma}_{C}(v) & =e^{-2 n \beta_{(\zeta, u)}\left(\left(0, u,|\zeta|^{2}\right),(0,0,1)\right)} d \mu_{(0,0,1)}(\zeta, u)=\frac{1}{4^{n}|\zeta|^{2 n}} d \zeta d u \\
& =\frac{1}{2^{2 n-1}|\zeta|^{4}} d \operatorname{vol}_{\mathbb{S}^{2 n-3}}\left(\frac{\zeta}{|\zeta|}\right) d u d\left(|\zeta|^{2}\right) .
\end{aligned}
$$

In particular,

$$
d \pi_{*} \widetilde{\sigma}_{C}\left(0, u,|\zeta|^{2}\right)=\frac{\operatorname{Vol}\left(\mathbb{S}^{2 n-3}\right)}{2^{2 n-1}} d u \frac{d\left(|\zeta|^{2}\right)}{|\zeta|^{4}}
$$

For every $x \in C$, let us denote by $\nu_{x}^{1} C$ the fiber over $x$ of the normal bundle map $v \mapsto \pi(v)$ from $\partial_{+}^{1} C$ to $C$, endowed with the spherical metric induced by the scalar product of the tangent space $T_{x} \mathbb{H}_{\mathbb{C}}^{n}$ at $x$. The Riemannian measure of $\partial_{+}^{1} C$ disintegrates under this fibration over the Riemannian measure of $C$ as

$$
d \operatorname{vol}_{\partial_{+}^{1} C}(v)=\int_{x \in C} d \operatorname{vol}_{\nu_{x}^{1} C}(v) d \operatorname{vol}_{C}(x) .
$$

Using the homeomorphism $\left(u, t=|\zeta|^{2}\right) \mapsto x=(0, u, t)$ from $\mathbb{R} \times[0,+\infty[$ to $C$, and Equation (8), we have

$$
d \operatorname{vol}_{C}(x)=\frac{d u d t}{4 t^{2}}=\frac{d u d\left(|\zeta|^{2}\right)}{4|\zeta|^{4}}
$$

Hence

$$
d \pi_{*} \operatorname{vol}_{\partial_{+}^{1} C}(x)=\operatorname{Vol}\left(\mathbb{S}^{2 n-3}\right) d \operatorname{vol}_{C}(x)=\frac{\operatorname{Vol}\left(\mathbb{S}^{2 n-3}\right)}{4} d u \frac{d\left(|\zeta|^{2}\right)}{|\zeta|^{4}} .
$$

The result follows as in the end of the proof of the previous Assertion.

By Theorem 7, we then have the following counting and equidistribution result of common perpendiculars. We first define some constants.

Let $D^{-}$be a horoball in $\mathbb{H}_{\mathbb{C}}^{n}$ centred at a parabolic fixed point of a lattice $\Gamma$ in $\operatorname{Isom}\left(\mathbb{H}_{\mathbb{C}}^{n}\right)$. If $D^{+}$is also a horoball in $\mathbb{H}_{\mathbb{C}}^{n}$ centred at a parabolic fixed point of $\Gamma$, let

$$
c\left(D^{-}, D^{+}\right)=\frac{4^{n} n !}{(2 n-1) \pi^{n}} \frac{\operatorname{Vol}\left(\Gamma_{D^{-}} \backslash D^{-}\right) \operatorname{Vol}\left(\Gamma_{D^{+}} \backslash D^{+}\right)}{\operatorname{Vol}(M)} .
$$

If $D^{+}$is a geodesic line in $\mathbb{H}_{\mathbb{C}}^{n}$ such that $\Gamma_{D^{+}} \backslash D^{+}$is compact, let

$$
c\left(D^{-}, D^{+}\right)=\frac{4^{n}(n !)^{2}}{(2 n) !(2 n-1) \pi} \frac{\operatorname{Vol}\left(\Gamma_{D^{-}} \backslash D^{-}\right) \operatorname{Vol}\left(\Gamma_{D^{+}} \backslash D^{+}\right)}{\operatorname{Vol}(M)} .
$$

If $D^{+}$is a complex geodesic line in $\mathbb{H}_{\mathbb{C}}^{n}$ such that $\Gamma_{D^{+}} \backslash D^{+}$has finite volume, let

$$
c\left(D^{-}, D^{+}\right)=\frac{4(n-1)}{(2 n-1) \pi} \frac{\operatorname{Vol}\left(\Gamma_{D^{-}} \backslash D^{-}\right) \operatorname{Vol}\left(\Gamma_{D^{+}} \backslash D^{+}\right)}{\operatorname{Vol}(M)} .
$$


Corollary 13 Let $\Gamma$ be a discrete group of isometries of $\mathbb{H}_{\mathbb{C}}^{n}$ such that the orbifold $M=$ $\Gamma \backslash \mathbb{H}_{\mathbb{C}}^{n}$ has finite volume. In each of the above three cases, if $m^{+}$is the cardinality of the pointwise stabiliser of $D^{+}$in $\Gamma$, then

$$
\mathscr{N}_{D^{-}, D^{+}}(t) \sim \frac{c\left(D^{-}, D^{+}\right)}{m^{+}} e^{2 n t} .
$$

If $\Gamma$ is arithmetic, then there exists $\kappa>0$ such that, as $t \rightarrow+\infty$,

$$
\mathscr{N}_{D^{-}, D^{+}}(t)=\frac{c\left(D^{-}, D^{+}\right)}{m^{+}} e^{2 n t}\left(1+\mathrm{O}\left(e^{-\kappa t}\right)\right) .
$$

Furthermore, if $D^{-}$is a horoball centred at a parabolic fixed point, then the origins of the common perpendiculars from $D^{-}$to the images of $D^{+}$under the elements of $\Gamma$ equidistribute in $\partial D^{-}$to the induced Riemannian measure:

$$
\frac{2 n m^{+} \operatorname{Vol}\left(\Gamma_{D^{-}} \backslash D^{-}\right)}{c\left(D^{-}, D^{+}\right)} e^{-2 n t} \sum_{x \in \partial D^{-}} m_{t}(x) \Delta_{x} \stackrel{*}{\rightarrow} \operatorname{vol}_{\partial D^{-}} .
$$

For smooth functions $\psi$ with compact support on $\partial D^{-}$, there is an error term in the equidistribution claim of Corollary 13 when the measures on both sides are evaluated on $\psi$, of the form $\mathrm{O}\left(e^{-\kappa t}\|\psi\|_{\ell}\right)$ where $\kappa>0$ and $\|\psi\|_{\ell}$ is the Sobolev norm of $\psi$ for some $\ell \in \mathbb{N}$, by the remark following Theorem 7

\section{A Mertens' formula for Heisenberg groups}

Let $K$ be an imaginary quadratic number field. We will denote, in Sections 5 to 7 , by $\mathscr{O}_{K}$ its ring of integers, by $D_{K}$ its discriminant, by $\zeta_{K}$ its zeta function, and by $\left|\mathscr{O}_{K}^{\times}\right|$the order of the unit group of $\mathscr{O}_{K}$. Let $\operatorname{tr}, \mathrm{n}: K \rightarrow \mathbb{Q}$ be the (absolute) trace and norm of $K$, that is $\operatorname{tr}(z)=z+\bar{z}=2 \operatorname{Re} z$ and $\mathrm{n}(z)=z \bar{z}=|z|^{2}$. We denote by $\langle a, \alpha, c\rangle$ the ideal of $\mathscr{O}_{K}$ generated by $a, \alpha, c \in \mathscr{O}_{K}$.

Let $\mathfrak{m}$ be a nonzero ideal in $\mathscr{O}_{K}$. We endow the ring $\mathscr{O}_{K} / \mathfrak{m}$ with the involution induced by the complex conjugation. Let $\mathrm{SU}_{q}\left(\mathscr{O}_{K} / \mathfrak{m}\right)$ be the finite group of $3 \times 3$ matrices in $\mathscr{O}_{K} / \mathfrak{m}$, having determinant 1 and preserving the Hermitian form $-z_{0} \overline{z_{2}}-z_{2} \overline{z_{0}}+z_{1} \overline{z_{1}}$ on $\left(\mathscr{O}_{K} / \mathfrak{m}\right)^{3}$. Let $B_{q}\left(\mathscr{O}_{K} / \mathfrak{m}\right)$ be its upper triangular subgroup. The action by shears on $\mathscr{O}_{K} \times \mathscr{O}_{K} \times \mathscr{O}_{K}$ of the nilpotent group $\operatorname{Heis}_{3}\left(\mathscr{O}_{K}\right)$ defined in the Introduction preserves $\mathscr{O}_{K} \times \mathfrak{m} \times \mathfrak{m}$. In this section, we will study the asymptotic of the counting function $\Psi_{\mathfrak{m}}$, where, for every $s \geq 0, \Psi_{\mathfrak{m}}(s)$ is the cardinality of

$$
\operatorname{Heis}_{3}\left(\mathscr{O}_{K}\right) \backslash\left\{(a, \alpha, c) \in \mathscr{O}_{K} \times \mathfrak{m} \times \mathfrak{m}: \operatorname{tr}(a \bar{c})=\mathrm{n}(\alpha),\langle a, \alpha, c\rangle=\mathscr{O}_{K}, \mathrm{n}(c) \leq s\right\} .
$$

When $\mathfrak{m}=\mathscr{O}_{K}$, this map $\Psi_{\mathfrak{m}}$ is the counting function, in terms of their standard heights, of the rational points over $K$ in the complex projective plane $\mathbb{P}_{2}(\mathbb{C})$, that lie in Segre's hyperconic with equation $2 \operatorname{Re} u-|v|^{2}=0$ in the standard affine chart with coordinates $(u, v)$.

Theorem 14 As $s \rightarrow+\infty$, we have

$$
\Psi_{\mathfrak{m}}(s)=\frac{\zeta(3)\left|B_{q}\left(\mathscr{O}_{K} / \mathfrak{m}\right)\right|}{2 \pi\left|D_{K}\right|^{\frac{1}{2}} \zeta_{K}(3)\left|\mathrm{SU}_{q}\left(\mathscr{O}_{K} / \mathfrak{m}\right)\right|} s^{2}+\mathrm{O}\left(s^{2-\kappa}\right) .
$$


The particular case $\mathfrak{m}=\mathscr{O}_{K}$ gives Theorem 4 in the Introduction. We will prove this result simultaneously with the next one. We endow the 3-dimensional Heisenberg group $\mathrm{Heis}_{3}$ with its Haar measure $\mathrm{Haar}_{\mathrm{Heis}_{3}}$ as in the introduction. The following result is an equidistribution result of the set of $\mathbb{Q}$-points (satisfying some congruence properties) in Heis $_{3}$. The particular case $\mathfrak{m}=\mathscr{O}_{K}$ gives Theorem 5 in the introduction.

Theorem 15 As $s \rightarrow+\infty$, we have

$$
\frac{\pi\left|D_{K}\right|^{\frac{3}{2}} \zeta_{K}(3)\left|\mathrm{SU}_{q}\left(\mathscr{O}_{K} / \mathfrak{m}\right)\right|}{\zeta(3)\left|B_{q}\left(\mathscr{O}_{K} / \mathfrak{m}\right)\right|} s^{-2} \sum_{\substack{(a, \alpha, c) \in \mathscr{O}_{K} \times \mathfrak{m} \times \mathfrak{m}, 0<\mathrm{n}(c) \leq s \\ \operatorname{tr}(a \bar{c})=\mathrm{n}(\alpha),\langle a, \alpha, c\rangle=\mathscr{O}_{K}}} \Delta_{\left(\frac{a}{c}, \frac{\alpha}{c}\right)} \stackrel{*}{\rightarrow} \text { Haar }_{\mathrm{Heis}_{3}} .
$$

As said after Corollary 13, for smooth functions $\psi$ with compact support on Heis 3 , there is an error term in this equidistribution result when the measures on both sides are evaluated on $\psi$, of the form $\mathrm{O}\left(s^{-\kappa}\|\psi\|_{\ell}\right)$ where $\kappa>0$ and $\|\psi\|_{\ell}$ is the Sobolev norm of $\psi$ for some $\ell \in \mathbb{N}$.

Proof of Theorem 14 and Theorem 15. As a preliminary remark, the 3-dimensional Heisenberg group Heis 3 defined above contains $\mathrm{Heis}_{3}\left(\mathscr{O}_{K}\right)$ (by the definition of the norm and trace of $K$ ), as a (uniform) lattice, and identifies with the Heisenberg group Heis3 defined in Section 3 by the change of variable

$$
(\zeta, u) \mapsto\left(w_{0}=\frac{1}{2}\left(|\zeta|^{2}-i u\right), w=\zeta\right)
$$

so that the Haar measures $\mathrm{Haar}_{\mathrm{Heis}_{3}}$ and $\lambda_{3}$ satisfy

$$
\lambda_{3}=2 \text { Haar }_{\mathrm{Heis}_{3}} .
$$

Let $q$ be the Hermitian form $-z_{0} \overline{z_{2}}-z_{2} \overline{z_{0}}+z_{1} \overline{z_{1}}$ of signature $(2,1)$ on $\mathbb{C} \times \mathbb{C} \times \mathbb{C}$ with coordinates $\left(z_{0}, z_{1}, z_{2}\right)$ (which is, up to isomorphism, the unique Hermitian form over $K$ with signature $(2,1)$ and determinant -1 , see [Sch, Ch. 10] for this cultural remark). As previously, we denote by $\mathrm{SU}_{q}$ the special unitary group of $q$. Let $\Gamma=\mathrm{SU}_{q} \cap \mathrm{SL}_{3}\left(\mathscr{O}_{K}\right)$ be the Picard modular group of $K$, which is a nonuniform arithmetic lattice in $\mathrm{SU}_{q}$ by a result of Borel and Harish-Chandra (see for instance [PaP1, §6.3]). As another cultural remark, every nonuniform arithmetic lattice in $\mathrm{SU}_{q}$ is commensurable to a Picard modular group (see for instance [Sto, $\S 3.1]$ ).

Consider the map from Heis 3 to $\mathrm{SU}_{q}$ defined, in the two sets of coordinates of Heis 3 defined in the Introduction and in Section 3 , by

$$
\left(w_{0}, w\right) \mapsto\left(\begin{array}{ccc}
1 & \bar{w} & w_{0} \\
0 & 1 & w \\
0 & 0 & 1
\end{array}\right) \quad \text { or } \quad(\zeta, u) \mapsto\left(\begin{array}{ccc}
1 & \bar{\zeta} & \frac{|\zeta|^{2}}{2}-i \frac{u}{2} \\
0 & 1 & \zeta \\
0 & 0 & 1
\end{array}\right) .
$$

This map is a Lie group isomorphism onto its image, by which we identify from now on Heis $_{3}$ and its image. Note that Heis $3 \cap \Gamma$ is then exactly $\operatorname{Heis}_{3}\left(\mathscr{O}_{K}\right)$.

We denote by $\Gamma_{\mathfrak{m}}$ the Hecke congruence subgroup of $\Gamma$ modulo $\mathfrak{m}$, that is the preimage, by the group morphism $\Gamma \rightarrow \mathrm{SL}_{3}\left(\mathscr{O}_{K} / \mathfrak{m}\right)$ of reduction modulo $\mathfrak{m}$, of the upper triangular subgroup of $\mathrm{SL}_{3}\left(\mathscr{O}_{K} / \mathfrak{m}\right)$. 
As previously, we denote by $\mathrm{PSU}_{q}$ the quotient Lie group $\mathrm{SU}_{q} /\left\{\mathrm{id}, j \mathrm{id}, j^{2}\right.$ id $\}$ where $j=e^{\frac{2 i \pi}{3}}$. For every subgroup $G$ of $\mathrm{SU}_{q}$, we denote by $\bar{G}$ its image in $\mathrm{PSU}_{q}$, and again by $g$ the image in $\mathrm{PSU}_{q}$ of any element $g$ of $\mathrm{SU}_{q}$.

We denote by $\Gamma_{\mathscr{H}_{1}}$ the stabiliser in $\Gamma_{\mathfrak{m}}$ of the horoball

$$
\mathscr{H}_{1}=\left\{\left[w_{0}: w: 1\right] \in \mathbb{P}_{2}(\mathbb{C}): 2 \operatorname{Re} w_{0}-|w|^{2} \geq 1\right\}
$$

centred at $\infty=[1: 0: 0]$ in the projective model of $\mathbb{H}_{\mathbb{C}}^{2}$. Note that this agrees with our notation for horoballs introduced in Section 3. The group $\Gamma_{\mathscr{H}_{1}}$ is independent of $\mathfrak{m}$, since $\Gamma_{\mathfrak{m}}$ is Hecke's congruence subgroup of $\Gamma$. Note that $\operatorname{Heis}_{3}\left(\mathscr{O}_{K}\right)$ is contained in $\Gamma_{\mathscr{H}_{1}}$, and that the projection map from $\operatorname{Heis}_{3}\left(\mathscr{O}_{K}\right)$ to $\overline{\operatorname{Heis}_{3}\left(\mathscr{O}_{K}\right)}$ is injective.

Let $B_{q}$ be as defined in Section 3 . Note that $\Gamma_{\mathscr{H}_{1}}=B_{q} \cap \Gamma_{\mathfrak{m}}=B_{q} \cap \Gamma$, since an isometry of $\mathbb{H}_{\mathbb{C}}^{2}$ fixes $\infty$ if and only if it preserves $\mathscr{H}_{1}$. We claim that the index of $\operatorname{Heis}_{3}\left(\mathscr{O}_{K}\right)$ in $\Gamma \mathscr{H}_{1}$ is

$$
\left[\Gamma_{\mathscr{H}_{1}}: \operatorname{Heis}_{3}\left(\mathscr{O}_{K}\right)\right]=\left|\mathscr{O}_{K}^{\times}\right|
$$

Indeed, it is the cardinality of the set of $\left(a_{1}, a_{2}, a_{3}\right) \in\left(\mathscr{O}_{K}^{\times}\right)^{3}$ such that $a_{1} a_{2} a_{3}=1, a_{3} \overline{a_{1}}=$ $1,\left|a_{2}\right|=1$, which is the cardinality of the set of $\left(a_{1}, a_{2}\right) \in\left(\mathscr{O}_{K}^{\times}\right)^{2}$ such that $a_{1}{ }^{2}=1 / a_{2}$. A separate treatment of the cases $D_{K}=-3,-4$ and of the general case gives the result. A similar argument shows that the projection map from $\Gamma_{\mathscr{H}_{1}}$ to $\overline{\Gamma_{\mathscr{H}}}$ is $\left(1+2 \delta_{D_{K},-3}\right)$-to-1 (in particular injective when $D_{K} \neq-3$ ).

For all $a, \alpha, c \in \mathscr{O}_{K}$ and $\lambda \in \mathbb{C}$, we have $\langle\lambda a, \lambda \alpha, \lambda c\rangle=\langle a, \alpha, c\rangle=\mathscr{O}_{K}$ if and only if $\lambda \in \mathscr{O}_{K}^{\times}$. Therefore the cardinality of the fibers of the projection map from $\{(a, \alpha, c) \in$ $\left.\mathscr{O}_{K} \times \mathfrak{m} \times \mathfrak{m}:\langle a, \alpha, c\rangle=\mathscr{O}_{K}\right\}$ to $\mathbb{P}_{2}(\mathbb{C})$ is equal to $\left|\mathscr{O}_{K}^{\times}\right|$, and no two distinct elements of such a fiber are sent one to the other by an element of $\mathrm{Heis}_{3}\left(\mathscr{O}_{K}\right)$. By [PaP1, Prop. 6.5 (2)], the orbit $\overline{\Gamma_{\mathfrak{m}}} \cdot \infty$ of $\infty=[1: 0: 0]$ under $\overline{\Gamma_{\mathfrak{m}}}$ is equal to the set of elements of $\mathbb{P}_{2}(\mathbb{C})$ which can be written in homogeneous coordinates $[a: \alpha: c]$ where $(a, \alpha, c) \in \mathscr{O}_{K} \times \mathfrak{m} \times \mathfrak{m}$, $\langle a, \alpha, c\rangle=\mathscr{O}_{K}$ and $\operatorname{tr}(a \bar{c})=\mathrm{n}(\alpha)$.

Let $g \in \mathrm{SU}_{q}$ be such that $g \mathscr{H}_{1}$ and $\mathscr{H}_{1}$ are disjoint (there are only finitely many double classes $[g] \in \overline{\Gamma_{\mathscr{H}}} \backslash \overline{\Gamma_{\mathfrak{m}}} / \overline{\Gamma_{\mathscr{H}}}$ for which this is not the case). If $\left(\begin{array}{c}a_{g} \\ \alpha_{g} \\ c_{g}\end{array}\right)$ is the first column of $g$, then $g \cdot \infty=\left[a_{g}: \alpha_{g}: c_{g}\right]$. Futhermore, by [PaP1, Lem. 6.3] and since the sectional curvature is normalised to have maximum -1 , the length of the common perpendicular $\delta_{g}$ between $g \mathscr{H}_{1}$ and $\mathscr{H}_{1}$ is then

$$
\ell\left(\delta_{g}\right)=\ln \left|c_{g}\right|-\ln 2 .
$$

We use, in the last one of the following equalities, Equation (21) and Corollary 13 with $n=2, \Gamma=\overline{\Gamma_{\mathfrak{m}}}$ and $D^{-}=D^{+}=\mathscr{H}_{1}$ (whose pointwise stabilisers in $\Gamma_{\mathfrak{m}}$ are trivial). We 
hence have, for some $\kappa>0$,

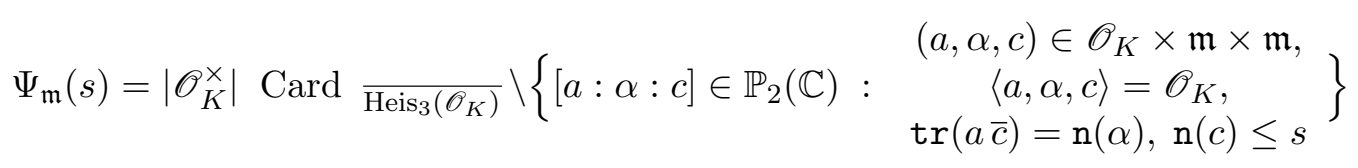

$$
\begin{aligned}
& =\left|\mathscr{O}_{K}^{\times}\right| \text {Card } \overline{\operatorname{Heis}_{3}\left(\mathscr{O}_{K}\right)} \backslash\left\{[a: \alpha: c] \in \overline{\Gamma_{\mathfrak{m}}} \cdot \infty: \begin{array}{l}
(a, \alpha, c) \in \mathscr{O}_{K} \times \mathfrak{m} \times \mathfrak{m}, \\
\langle a, \alpha, c\rangle=\mathscr{O}_{K}, \mathrm{n}(c) \leq s
\end{array}\right\} \\
& =\left|\mathscr{O}_{K}^{\times}\right| \text {Card }\left\{[g] \in \overline{\operatorname{Heis}_{3}\left(\mathscr{O}_{K}\right)} \backslash \overline{\Gamma_{\mathfrak{m}}} / \overline{\Gamma_{\mathscr{H}}}: \mathrm{n}\left(c_{g}\right) \leq s\right\} \\
& =\left|\mathscr{O}_{K}^{\times}\right|\left[\overline{\Gamma_{\mathscr{H}_{1}}}: \overline{\operatorname{Heis}_{3}\left(\mathscr{O}_{K}\right)}\right] \operatorname{Card}\left\{[g] \in \overline{\Gamma_{\mathscr{H}_{1}}} \backslash \overline{\Gamma_{\mathfrak{m}}} / \overline{\Gamma_{\mathscr{H}}}: \ell\left(\delta_{g}\right) \leq \frac{\ln s}{2}-\ln 2\right\}+\mathrm{O}(1) \\
& =\left|\mathscr{O}_{K}^{\times}\right| \frac{\left[\Gamma_{\mathscr{H}_{1}}: \operatorname{Heis}_{3}\left(\mathscr{O}_{K}\right)\right]}{1+2 \delta_{D_{K},-3}} \mathscr{N}_{\mathscr{H}_{1}, \mathscr{H}_{1}}\left(\frac{\ln s}{2}-\ln 2\right)+\mathrm{O}(1) \\
& =\frac{2\left|\mathscr{O}_{K}^{\times}\right|^{2} \operatorname{Vol}\left(\overline{\Gamma_{\mathscr{H}_{1}}} \backslash \mathscr{H}_{1}\right)^{2}}{3 \pi^{2}\left(1+2 \delta_{D_{K},-3}\right) \operatorname{Vol}\left(\overline{\Gamma_{\mathfrak{m}}} \backslash \mathbb{H}_{\mathbb{C}}^{2}\right)} s^{2}\left(1+\mathrm{O}\left(s^{-\kappa}\right)\right)
\end{aligned}
$$

The next two lemmas are devoted to the computation of the two volumes that appear in the previous line.

Lemma 16 We have $\operatorname{Vol}\left(\overline{\Gamma_{\mathscr{H}_{1}}} \backslash \mathscr{H}_{1}\right)=\frac{\left(1+2 \delta_{D_{K},-3}\right)\left|D_{K}\right|}{8\left|\mathscr{O}_{K}^{\times}\right|}$.

Proof. We follow arguments similar to the ones in the reference [KK, §4], which uses the same convention as [Par2] for the Riemannian measure on Heis $2 n-1$, see also [FaP, $\S 3.1]$ when $D_{K}=-3$. Let $n=2$. As in [Par2], we endow Heis 3 with the left-invariant Riemannian metric

$$
(d u+2 \operatorname{Im}(d \zeta \bar{\zeta}))^{2}+4 d \zeta \overline{d \zeta}
$$

whose Riemannian volume is $\operatorname{vol}_{\text {Heis } 3}=4 \lambda_{3}$. In particular, the Heis 3 -equivariant map from $\partial \mathscr{H}_{1}$ to Heis 3 , which in horospherical coordinates maps $(\zeta, u, t)$ to $(\zeta, u)$, sends $\operatorname{vol}_{\partial \mathscr{H}_{1}}$ to $\frac{1}{2} \lambda_{3}=\frac{1}{8} \mathrm{vol}_{\mathrm{Heis}}$, by Equation (8).

Let $t_{K}$ be the minimal vertical translation in $\operatorname{Heis}_{3}\left(\mathscr{O}_{K}\right)$, that is, the minimal $s>0$ such that $\left(w_{0}=\frac{i s}{2}, w=0\right) \in \operatorname{Heis}_{3}\left(\mathscr{O}_{K}\right)$. In particular,

$$
t_{K}=\min \left\{s>0: \frac{i s}{2} \in \mathscr{O}_{K}\right\}
$$

Recall that $\mathscr{O}_{K}=\mathbb{Z}+\mathbb{Z} \frac{i \sqrt{\left|D_{K}\right|}}{2}$ if $D_{k} \equiv 0 \bmod 4$ and $\mathscr{O}_{K}=\mathbb{Z}+\mathbb{Z} \frac{1+i \sqrt{\left|D_{K}\right|}}{2}$ if $D_{k} \equiv 1$ $\bmod 4$. Hence $t_{K}=\sqrt{\left|D_{K}\right|}$ if $D_{k} \equiv 0 \bmod 4$ and $t_{K}=2 \sqrt{\left|D_{K}\right|}$ if $D_{k} \equiv 1 \bmod 4$.

Consider the following set

$$
\begin{aligned}
\Pi\left(\operatorname{Heis}_{3}\left(\mathscr{O}_{K}\right)\right) & =\left\{w \in \mathbb{C}: \exists w_{0} \in \mathbb{C},\left(w_{0}, w\right) \in \operatorname{Heis}_{3}\left(\mathscr{O}_{K}\right)\right\} \\
& =\left\{w \in \mathscr{O}_{K}: \exists w_{0} \in \mathscr{O}_{K}, \operatorname{tr}\left(w_{0}\right)=\mathrm{n}(w)\right\}=\mathrm{n}^{-1}\left(\operatorname{tr}\left(\mathscr{O}_{K}\right)\right) .
\end{aligned}
$$

We have $\operatorname{tr}\left(\mathscr{O}_{K}\right)=\mathbb{Z}$ if $D_{K} \equiv 1 \bmod 4$. If $D_{K} \equiv 0 \bmod 4$, then $\operatorname{tr}\left(\mathscr{O}_{K}\right)=2 \mathbb{Z}$ and for all $a, b \in \mathbb{Z}$, the integer $\mathrm{n}\left(a+b i \frac{\sqrt{\left|D_{K}\right|}}{2}\right)=a^{2}+b^{2} \frac{\left|D_{K}\right|}{4}$ is even if and only if

- $a$ is even, when $\frac{\left|D_{K}\right|}{4}$ is even,

- $a-b$ is even, when $\frac{\left|D_{K}\right|}{4}$ is odd. 
Hence $\Pi\left(\operatorname{Heis}_{3}\left(\mathscr{O}_{K}\right)\right)$ is a $\mathbb{Z}$-sublattice in $\mathscr{O}_{K}$ with index equal to 1 if $D_{K} \equiv 1 \bmod 4$ and equal to 2 if $D_{K} \equiv 0 \bmod 4$.

By [Par2, Lem. 1.2], we have

$$
\operatorname{Vol}_{\mathrm{Heis}_{3}}\left(\operatorname{Heis}_{3}\left(\mathscr{O}_{K}\right) \backslash \operatorname{Heis}_{3}\right)=4 t_{K} \operatorname{Vol}\left(\mathbb{C} / \Pi\left(\operatorname{Heis}_{3}\left(\mathscr{O}_{K}\right)\right)\right)
$$

Note that $\operatorname{Vol}\left(\mathbb{C} / \mathscr{O}_{K}\right)=\frac{\sqrt{\left|D_{K}\right|}}{2}$ since $\mathscr{O}_{K}$ is generated as a $\mathbb{Z}$-module by 1 and $\left(D_{K}+\right.$ $\left.i \sqrt{\left|D_{K}\right|}\right) / 2$. Hence

$$
\left.\operatorname{Vol}_{\text {Heis } 3}\left(\operatorname{Heis}_{3}\left(\mathscr{O}_{K}\right) \backslash \operatorname{Heis}_{3}\right)=4 t_{K}\left[\mathscr{O}_{K}: \Pi\left(\operatorname{Heis}_{3}\left(\mathscr{O}_{K}\right)\right)\right] \operatorname{Vol}\left(\mathbb{C} / \mathscr{O}_{K}\right)\right)=4\left|D_{K}\right|
$$

As already seen, the map $\Gamma_{\mathscr{H}_{1}} \rightarrow \overline{\Gamma_{\mathscr{H}_{1}}}$ is a 3 -to- 1 map if $D_{K}=-3$ and injective otherwise. By Lemma 11, we then have

$$
\begin{aligned}
& \operatorname{Vol}\left(\overline{\Gamma_{\mathscr{H}}} \backslash \mathscr{H}_{1}\right)=\frac{1}{4} \operatorname{Vol}\left(\overline{\Gamma_{\mathscr{H}}} \backslash \partial \mathscr{H}_{1}\right) \\
& =\frac{1}{4\left[\overline{\Gamma_{\mathscr{H}_{1}}}: \overline{\operatorname{Heis}_{3}\left(\mathscr{O}_{K}\right)}\right]} \operatorname{Vol}\left(\overline{\operatorname{Heis}_{3}\left(\mathscr{O}_{K}\right)} \backslash \partial \mathscr{H}_{1}\right) \\
& =\frac{1+2 \delta_{D_{K},-3}}{32\left[\Gamma_{\mathscr{H}_{1}}: \operatorname{Heis}_{3}\left(\mathscr{O}_{K}\right)\right]} \operatorname{Vol}_{\mathrm{Heis}_{3}}\left(\operatorname{Heis}_{3}\left(\mathscr{O}_{K}\right) \backslash \operatorname{Heis}_{3}\right) \text {. }
\end{aligned}
$$

The result hence follows from Equation (21) and Equation (24).

The volume of the orbifold $\overline{\Gamma_{\mathfrak{m}}} \backslash \mathbb{H}_{\mathbb{C}}^{2}$ is known (see for instance [Hol3, Sto] ). We only give a proof since the normalisation of the measures is a bit tricky to follow amongst the various references.

Lemma 17 We have $\operatorname{Vol}\left(\overline{\Gamma_{\mathfrak{m}}} \backslash \mathbb{H}_{\mathbb{C}}^{2}\right)=\frac{\left(1+2 \delta_{D_{K},-3}\right)\left|D_{K}\right|^{5 / 2} \zeta_{K}(3)\left|\mathrm{SU}_{q}\left(\mathscr{O}_{K} / \mathfrak{m}\right)\right|}{48 \pi \zeta(3)\left|B_{q}\left(\mathscr{O}_{K} / \mathfrak{m}\right)\right|}$.

Proof. In the complex ball

$$
\mathbb{B}_{\mathbb{C}}^{2}=\left\{\left(z_{1}=x_{1}+i y_{1}, z_{2}=x_{2}+i y_{2}\right) \in \mathbb{C}^{2}:\left|z_{1}\right|^{2}+\left|z_{2}\right|^{2}<1\right\},
$$

the Riemannian metric invariant under its group of biholomorphisms, which makes it isometric to the Siegel domain $\mathbb{H}_{\mathbb{C}}^{2}$, has volume form at the point $(0,0)$ equal to

$$
4 d x_{1} \wedge d y_{1} \wedge d x_{2} \wedge d y_{2}
$$

(see [Gol, page 105], which normalises the sectional curvature to be in $\left[-1,-\frac{1}{4}\right]$, as we normalise it to be in $[-4,-1]$ in this paper). The volume form $\operatorname{vol}_{\mathrm{Hol}}$ at the point $(0,0)$ of $\mathbb{B}_{\mathbb{C}}^{2}$ used in [Hol3, page 86] for its volume computation is

$$
\frac{6}{\pi^{2}} d x_{1} \wedge d y_{1} \wedge d x_{2} \wedge d y_{2} .
$$

The Cayley transform from the Siegel domain to the ball model of the complex hyperbolic space conjugates $\bar{\Gamma}$ to the Picard modular group $\Gamma$ used by Holzapfel in loc. cit.. Hence $\operatorname{Vol}\left(\bar{\Gamma} \backslash \mathbb{H}_{\mathbb{C}}^{2}\right)=\frac{2 \pi^{2}}{3} \operatorname{Vol}_{\mathrm{Hol}}\left(\Gamma \backslash \mathbb{B}_{\mathbb{C}}^{2}\right)$. By the Main Theorem 4.9 of [Hol3, page 83], we have

$$
\operatorname{Vol}_{\mathrm{Hol}}\left(\Gamma \backslash \mathbb{B}_{\mathbb{C}}^{2}\right)=\frac{\left(1+2 \delta_{D_{K},-3}\right)\left|D_{K}\right|^{5 / 2} L_{K}(3)}{32 \pi^{3}},
$$


where $L_{K}(s)=\sum_{n=1}^{\infty} \chi_{K}(n) / n^{s}$ is Dirichlet's $L$-series of $K$, whose character is given by Jacobi's symbol $\chi_{K}(n)=\left(\frac{D_{K}}{n}\right)$. Hence, using the well known relation $L_{K}(s)=\zeta_{K}(s) / \zeta(s)$ between Dirichlet's $L$-series and Dedekind's zeta function of $K$, the result follows since

$$
\left[\bar{\Gamma}: \overline{\Gamma_{\mathfrak{m}}}\right]=\left[\Gamma: \Gamma_{\mathfrak{m}}\right]=\left[\mathrm{SU}_{q}\left(\mathscr{O}_{K} / \mathfrak{m}\right): B_{q}\left(\mathscr{O}_{K} / \mathfrak{m}\right)\right]=\frac{\left|\mathrm{SU}_{q}\left(\mathscr{O}_{K} / \mathfrak{m}\right)\right|}{\left|B_{q}\left(\mathscr{O}_{K} / \mathfrak{m}\right)\right|}
$$

Theorem 14 follows from Lemma 17, Lemma 16 and Equation (23).

Let us prove now Theorem 15 .

The orthogonal projection map $f: \partial_{\infty} \mathbb{H}_{\mathbb{C}}^{2}-\{\infty\} \rightarrow \partial \mathscr{H}_{1}$ is the homeomorphism defined by $\left[w_{0}: w: 1\right] \mapsto\left(\zeta=w, u=-2 \operatorname{Im} w_{0}, 1\right)$ using the homogeneous coordinates on $\partial_{\infty} \mathbb{H}_{\mathbb{C}}^{2}-\{\infty\}$ and the horospherical coordinates on $\partial \mathscr{H}_{1}$ (see Equation $(7)$ ). Let $x \in \partial \mathscr{H}_{1}$ be the origin of a common perpendicular of length at most $t$ from $\mathscr{H}_{1}$ to an element $\gamma \mathscr{H}_{1}$ for some $\gamma \in \Gamma_{\mathfrak{m}}$ not fixing $\infty$. By the previous arguments, $x$ is the orthogonal projection on $\mathscr{H}_{1}$ of the point at infinity of this horoball $\gamma \mathscr{H}_{1}$. This point at infinity may be written $\left[\frac{a}{c}: \frac{\alpha}{c}: 1\right]$ for some triple $(a, \alpha, c) \in \mathscr{O}_{K} \times \mathfrak{m} \times \mathfrak{m}$ with $\langle a, \alpha, c\rangle=\mathscr{O}_{K}, \operatorname{tr}(a \bar{c})=\mathrm{n}(\alpha)$ and $0<\mathrm{n}(c) \leq 4 e^{2 t}$ (using Equation $(22)$ ). There are exactly $\left|\mathscr{O}_{K}^{\times}\right|$such triples. Hence by Equation (19), considering the value of $C\left(D^{-}, D^{+}\right)$for $D^{-}=D^{+}=\mathscr{H}_{1}$, using the horospherical coordinates on $\partial \mathscr{H}_{1}$, we have, as $t \rightarrow+\infty$,

$$
\frac{3 \pi^{2} \operatorname{Vol}\left(\overline{\Gamma_{\mathfrak{m}}} \backslash \mathbb{H}_{\mathbb{C}}^{2}\right)}{8 \operatorname{Vol}\left(\overline{\Gamma_{\mathscr{H}}} \backslash \mathscr{H}_{1}\right)\left|\mathscr{O}_{K}^{\times}\right|} e^{-4 t} \sum_{\substack{(a, \alpha, c) \in \mathscr{O}_{K} \times \mathfrak{m} \times \mathfrak{m}, 0<\mathrm{n}(c) \leq 4 e^{2 t} \\ \operatorname{tr}(a \bar{c})=\mathrm{n}(\alpha),\langle a, \alpha, c)=\mathscr{O}_{K}}} \Delta_{\left(\frac{\alpha}{c},-2 \operatorname{Im} \frac{a}{c}, 1\right)} \stackrel{*}{\rightarrow} \operatorname{vol}_{\partial \mathscr{H}_{1}}
$$

The image of the Haar measure Haar Heis $_{3}$ (defined in Equation (2)) by $f$ is, by Equation (8),

$$
f_{*} \operatorname{Haar}_{\text {Heis }}=\operatorname{vol}_{\partial \mathscr{H}_{1}} .
$$

Using the change of variables $s=4 e^{2 t}$, the identification of $\partial_{\infty} \mathbb{H}_{\mathbb{C}}^{2}-\{\infty\}$ with Heis 3 and the continuity of the pushforward by $f^{-1}$ of the measures on $\partial \mathscr{H}_{1}$ applied to Equation 25), we hence have, as $s \rightarrow+\infty$,

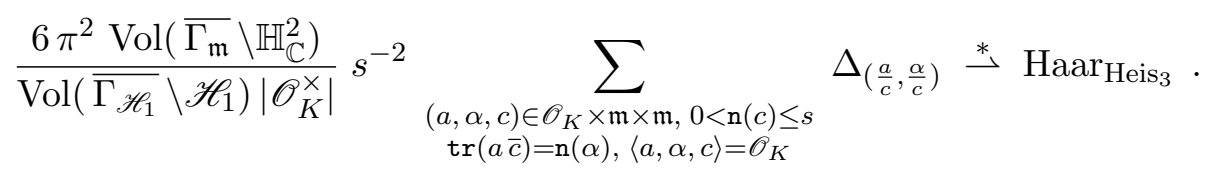

Finally, Theorem 15 follows from this and from Lemma 17 and Lemma 16.

Remark. A result of Feustel [Feu] (see also [Hol2, page 280] and [Zin]) says that the map, which associates to a parabolic fixed point of $\bar{\Gamma}$ the fractional ideal generated by its homogeneous coordinates in $\mathscr{O}_{K}$, induces a bijection from the set of cusps (that is, of orbits under $\bar{\Gamma}$ of its parabolic fixed points) to the set of ideal classes of $K$. In Theorem 4 and Theorem 5, replacing in its proof $D^{+}=\mathscr{H}_{1}$ by a horoball centred at a parabolic fixed point $p$ of $\bar{\Gamma}$ not in the orbit of $\infty$ (which hence exists if and only if $\left.D_{K} \neq-3,-4,-7,-8,-11,-19,-43,-67,-163\right)$, we can obtain a counting and equidistribution result with error term in Heis 3 of the points in $\Gamma \cdot p$. But the volume of the quotient of this new $D^{+}$by its stabiliser in $\bar{\Gamma}$ is not explicit for the moment, hence we would not have results as precise as in the case $p=\infty$. 
Remark 18 Theorem 14 and Theorem 15 have generalisations in higher dimension. Let $n \geq 2$, and let $\left(w, w^{\prime}\right) \mapsto w \cdot \overline{w^{\prime}}$ be the standard Hermitian scalar product on $\mathbb{C}^{n-1}$. Let

$$
\text { Heis }_{2 n-1}=\left\{\left(w_{0}, w\right) \in \mathbb{C} \times \mathbb{C}^{n-1}: 2 \operatorname{Re} w_{0}=w \cdot \bar{w}\right\},
$$

with law $\left(w_{0}, w\right)\left(w_{0}^{\prime}, w^{\prime}\right)=\left(w_{0}+w_{0}^{\prime}+w^{\prime} \cdot \bar{w}, w+w^{\prime}\right)$, be the Heisenberg group of dimension $2 n-1$, which identifies with the boundary at infinity of the Siegel domain $\mathbb{H}_{\mathbb{C}}^{n}$ with $\infty$ removed. Let $q$ be the Hermitian form defined in Section 3 . Let $\mathrm{SU}_{q}$ be its special unitary group and $\Gamma=\mathrm{SU}_{q} \cap \mathscr{M}_{n}\left(\mathscr{O}_{K}\right)$, which is an arithmetic lattice in $\mathrm{SU}_{q}$. Then Corollary 13 (which is valid in any dimension), applied with the image $\bar{\Gamma}$ of $\Gamma$ in $\mathrm{PSU}_{q}$ and with $D^{-}=D^{+}$the horoball of points in $\mathbb{H}_{\mathbb{C}}^{n}$ with last horospherical coordinates at least 1 , gives a counting and equidistribution result with error term in Heis ${ }_{2 n-1}$ of the points in $\bar{\Gamma} \cdot \infty-\{\infty\}$. The volume of $\bar{\Gamma} \backslash \mathbb{H}_{\mathbb{C}}^{n}$ could be computed using [EmS], up to computing the index of $\Gamma$ in a principal arithmetic subgroup containing it. But the volume of the cusp corresponding to $\infty$ in $\bar{\Gamma} \backslash \mathbb{H}_{\mathbb{C}}^{n}$ is not explicit for the moment, hence we would not have results as precise as in the case $n=2$.

Other counting and equidistribution results of arithmetically defined points in the Heisenberg group Heis $_{2 n-1}$ may be obtained by varying the integral Hermitian form $q$ of signature $(1, n)$ and the arithmetic lattice $\Gamma$ in $\mathrm{SU}_{q}$.

\section{Counting cubic points over quadratic imaginary fields in the projective plane}

Let $K$ be an imaginary quadratic number field. Let $q$ be the Hermitian form $-z_{0} \overline{z_{2}}-$ $z_{2} \overline{z_{0}}+\left|z_{1}\right|^{2}$ on $\mathbb{C}^{3}$ (the following result could be adapted to any Hermitian form on $K^{3}$ with complex signature $(1,2)$, see for instance [Sch, Ex. 1.6(iv), p. 351] for their classification). We will say that a point in the complex projective plane $\mathbb{P}_{2}(\mathbb{C})$ is isotropic (respectively that two projective points are orthogonal) if the corresponding complex lines in $\mathbb{C}^{3}$ are isotropic (respectively orthogonal) for $q$.

The Galois group $\operatorname{Gal}(\mathbb{C} \mid K)$ acts naturally on $\mathbb{P}_{2}(\mathbb{C})$ by $\sigma\left[z_{0}: z_{1}: z_{2}\right]=\left[\sigma z_{0}: \sigma z_{1}: \sigma z_{2}\right]$ using homogeneous coordinates. A point $z \in \mathbb{P}_{2}(\mathbb{C})$ will be called Hermitian cubic over $K$ if it is cubic over $K$ (that is, if its orbit under $\operatorname{Gal}(\mathbb{C} \mid K)$ has exactly three points), and if its other conjugates $z^{\prime}, z^{\prime \prime}$ over $K$ are isotropic and orthogonal to $z$.

We will denote by $(\gamma, z) \mapsto \gamma \cdot z$ the projective action of $\mathrm{SL}_{3}(\mathbb{C})$ or $\mathrm{PSL}_{3}(\mathbb{C})$ on $\mathbb{P}_{2}(\mathbb{C})$. Recall that $\mathrm{SU}_{q}$ is the real Lie group of linear automorphisms of $\mathbb{C}^{3}$ having determinant 1 and preserving $q$. Let $\Gamma=\mathrm{SU}_{q}\left(\mathscr{O}_{K}\right)=\mathrm{SU}_{q} \cap \mathrm{SL}_{3}\left(\mathscr{O}_{K}\right)$, which is an arithmetic lattice in $\mathrm{SU}_{q}$. The action of $\Gamma$ on $\mathbb{P}_{2}(\mathbb{C})$ preserves the sets of rational, cubic and Hermitian cubic points of $\mathbb{P}_{2}(\mathbb{C})$ over $K$. The number of orbits of Hermitian cubic points is infinite, which explains why we will restrict ourselves to a given orbit below. Let $\Gamma_{\infty}$ be the stabiliser of $\infty=[1: 0: 0]$ in $\Gamma$, which preserves the Cygan distance $d_{\mathrm{Cyg}}$ and its modifications $d_{\text {Cyg }}^{\prime}, d_{\text {Cyg }}^{\prime \prime}$ on $\mathrm{Heis}_{3}=\left\{\left[w_{0}: w: 1\right] \in \mathbb{P}_{2}(\mathbb{C}): 2 \operatorname{Re} w_{0}=|w|^{2}\right\}$.

Let $z \in \mathbb{P}_{2}(\mathbb{C})$ be Hermitian cubic over $K$, and let $z^{\prime}, z^{\prime \prime}$ be its other conjugates over $K$. Since $z^{\prime}, z^{\prime \prime}$ are distinct, irrational over $K$, and isotropic for $q$, they lie in Heis 3 , and we may define the complexity $c(z)$ of $z$ as the inverse of a modification of the Cygan distance between its conjugates over $K$ :

$$
c(z)=d_{\text {Cyg }}^{\prime \prime}\left(z^{\prime}, z^{\prime \prime}\right)^{-1} .
$$


The complexity of $z$ is in particular invariant under $\Gamma_{\infty}$. It is part of the proof of the following result that the number of projective points, that are Hermitian cubic over $K$, belong to a given orbit of $\Gamma=\mathrm{SU}_{q}\left(\mathscr{O}_{K}\right)$, and have complexity at most $s$, is finite for every $s \geq 0$. It turns out that the use of $d_{\mathrm{Cyg}}^{\prime \prime}$ instead of the actual Cygan distance $d_{\text {Cyg }}$ allows in this Section to give precise asymptotic results with error terms, instead of upper/lower estimates that differ by a multiplicative constant.

Let us now state and prove an asymptotic estimate as $s \rightarrow+\infty$ of the counting function of the set of points in the projective plane that are Hermitian cubic over $K$, in a given orbit of for instance a congruence subgroup of $\mathrm{SU}_{q}\left(\mathscr{O}_{K}\right)$, with complexity at most $s$.

Theorem 19 Let $K$ be an imaginary quadratic number field. Let $z_{0} \in \mathbb{P}_{2}(\mathbb{C})$ be Hermitian cubic over $K$. Let $G$ be a finite index subgroup of $\operatorname{PSU}_{q}\left(\mathscr{O}_{K}\right)$, and let $G_{\infty}$ be the stabiliser of $\infty=[1: 0: 0]$ in $G$. Then there exists $\kappa>0$ such that, as $s \rightarrow+\infty$,

$$
\begin{aligned}
\operatorname{Card}\left\{z \in G_{\infty} \backslash G \cdot z_{0}:\right. & c(z) \leq s\} \\
& =\frac{256 \ln \left|\lambda_{0}\right| \zeta(3)\left[\operatorname{PSU}_{q}\left(\mathscr{O}_{K}\right)_{\infty}: G_{\infty}\right]}{3 \iota_{0} n_{0}\left|\mathscr{O}_{K}^{\times}\right|\left|D_{K}\right|^{3 / 2} \zeta_{K}(3)\left[\operatorname{PSU}_{q}\left(\mathscr{O}_{K}\right): G\right]} s^{4}\left(1+\mathrm{O}\left(s^{-\kappa}\right)\right)
\end{aligned}
$$

where $\left|\lambda_{0}\right|$ is the smallest modulus $>1$ of an eigenvalue of an element in $G$ fixing the other two conjugates $z_{0}^{\prime}, z_{0}^{\prime \prime}$ of $z_{0}$ over $K$, where $\iota_{0}=2$ if $G$ contains an element exchanging $z_{0}^{\prime}$ and $z_{0}^{\prime \prime}$ and $\iota_{0}=1$ otherwise, and where $n_{0}$ is the cardinality of the pointwise stabiliser in $G$ of the projective line through $z_{0}^{\prime}$ and $z_{0}^{\prime \prime}$.

Proof. The group $G$, which has finite index in the arithmetic lattice $\bar{\Gamma}=\operatorname{PSU}_{q}\left(\mathscr{O}_{K}\right)$, is also an arithmetic discrete group of isometries with finite covolume in the complex hyperbolic plane $\mathbb{H}_{\mathbb{C}}^{2}$. By Lemma 17 , we have

$$
\begin{aligned}
\operatorname{Vol}\left(G \backslash \mathbb{H}_{\mathbb{C}}^{2}\right) & =[\bar{\Gamma}: G] \operatorname{Vol}\left(\bar{\Gamma} \backslash \mathbb{H}_{\mathbb{C}}^{2}\right) \\
& =\frac{[\bar{\Gamma}: G]\left(1+2 \delta_{D_{K},-3}\right)\left|D_{K}\right|^{5 / 2} \zeta_{K}(3)}{48 \pi \zeta(3)}
\end{aligned}
$$

Let $n_{\infty}=\left[\bar{\Gamma}_{\infty}: G_{\infty}\right]$ be the index of $G_{\infty}$ in the stabiliser of $\infty$ in $\bar{\Gamma}$. By Lemma 16 , since $G_{\infty}$ is equal to the stabiliser $G_{\mathscr{H}_{1}}$ in $G$ of the horosphere $\mathscr{H}_{1}$, we have

$$
\operatorname{Vol}\left(G_{\infty} \backslash \mathscr{H}_{1}\right)=\operatorname{Vol}\left(G_{\mathscr{H}_{1}} \backslash \mathscr{H}_{1}\right)=\frac{n_{\infty}\left(1+2 \delta_{D_{K},-3}\right)\left|D_{K}\right|}{2\left|\mathscr{O}_{K}^{\times}\right|}
$$

Proposition 20 A point $z_{0} \in \mathbb{P}_{2}(\mathbb{C})$ is Hermitian cubic over $K$ if and only if there exists $\gamma_{0} \in \operatorname{PSU}_{q}\left(\mathscr{O}_{K}\right)$ of infinite order and $K$-irreducible such that $z_{0}$ is the only fixed point of $\gamma_{0}$ that belongs to the positive cone of $q$ in $\mathbb{P}_{2}(\mathbb{C})$.

Recall that an element of $\operatorname{PSU}_{q}(K)$ is $K$-irreducible if it does not preserve a point or a line defined over $K$ in $\mathbb{P}_{2}(\mathbb{C})$.

Proof. (Y. Benoist) Assume first that $\gamma_{0} \in \operatorname{PSU}_{q}\left(\mathscr{O}_{K}\right)$ has infinite order and is $K$ irreducible. Then $\gamma_{0}$ is not an elliptic element, since elliptic elements of $\operatorname{PSU}_{q}\left(\mathscr{O}_{K}\right)$ have finite order. It is not parabolic, since the fixed points in $\partial_{\infty} \mathbb{H}_{\mathbb{C}}^{2}$ of the parabolic elements of $\operatorname{PSU}_{q}\left(\mathscr{O}_{K}\right)$ are rational over $K$ (see [Hol1] or [Hol2, page 290]). Hence, it is loxodromic, and fixes exactly two distinct points $z_{0}^{\prime}$ and $z_{0}^{\prime \prime}$ in $\partial_{\infty} \mathbb{H}_{\mathbb{C}}^{2}$. In particular, $z_{0}^{\prime}$ and $z_{0}^{\prime \prime}$ are 
isotropic for $q$. Since $\gamma_{0}$ belongs to $\operatorname{PSL}_{3}(\mathbb{C})$ and preserves $\partial_{\infty} \mathbb{H}_{\mathbb{C}}^{2}$, it preserves the unique complex projective lines $L^{\prime}$ and $L^{\prime \prime}$ tangent to $\partial_{\infty} \mathbb{H}_{\mathbb{C}}^{2}$ at the points $z_{0}^{\prime}$ and $z_{0}^{\prime \prime}$, respectively. Note that $L^{\prime}$ and $L^{\prime \prime}$ are exactly the sets of points in $\mathbb{P}_{2}(\mathbb{C})$ which are orthogonal to $z_{0}^{\prime}$ and $z_{0}^{\prime \prime}$, respectively. The projective lines $L^{\prime}$ and $L^{\prime \prime}$ meet at exactly one point $z_{0}$, which belongs to the complement in $\mathbb{P}_{2}(\mathbb{C})$ of $\mathbb{H}_{\mathbb{C}}^{2} \cup \partial_{\infty} \mathbb{H}_{\mathbb{C}}^{2}$. This complement is exactly the positive cone of $q$ in $\mathbb{P}_{2}(\mathbb{C})$. The fixed points $z_{0}, z_{0}^{\prime}, z_{0}^{\prime \prime}$ of $\gamma_{0}$ are at most cubic over $K$, since $\gamma_{0}$ has coefficients in $K$. They are exactly cubic and conjugates, since $\gamma_{0}$ is $K$-irreducible. Hence $z_{0}$ is Hermitian cubic, and is the only fixed point of $\gamma_{0}$ in the positive cone of $q$.

Conversely, let $z_{0} \in \mathbb{P}_{2}(\mathbb{C})$ be Hermitian cubic over $K$, and let $z_{0}^{\prime}, z_{0}^{\prime \prime}$ be its other two Galois conjugates. Let $G$ be the linear algebraic group defined over $\mathbb{Q}$, such that $\underline{G}(\mathbb{Z})=\operatorname{PSU}_{q}\left(\mathscr{O}_{K}\right)$ and $\underline{G}(\mathbb{R})=\mathrm{PSU}_{q}$. It has $\mathbb{R}$-rank one. The pointwise stabiliser of $\left\{z_{0}^{\prime}, z_{0}^{\prime \prime}\right\}$ in $\underline{G}$ is the centraliser $Z(\underline{T})$ of a maximal algebraic torus $\underline{T}$ in $\underline{G}$, since $z_{0}^{\prime}, z_{0}^{\prime \prime}$ are distinct and isotropic. Note that $Z(\underline{T})$ also fixes $z_{0}$, since $z_{0}$, being orthogonal to $z_{0}^{\prime}$ and $z_{0}^{\prime \prime}$, belongs to the complex projective lines tangent to the null cone of $q$ in $\mathbb{P}_{2}(\mathbb{C})$ at $z_{0}^{\prime}$ and $z_{0}^{\prime \prime}$, and as seen above, these two projective lines meet at exactly one point. The algebraic group $Z(\underline{T})$, being the pointwise stabiliser of $\left\{z_{0}, z_{0}^{\prime}, z_{0}^{\prime \prime}\right\}$ which is invariant under $\operatorname{Gal}(\mathbb{C} / K)$, is defined over $K$. The torus $\underline{T}$ has rank one and has finite index in $Z(\underline{T})$. Hence $\underline{T}$ is also defined over $K$, and is isomorphic to $\mathbb{C}^{\times}$over $\mathbb{C}$. It has no nontrivial $\mathbb{Q}$-character (as it is one-dimensional, it is not defined over $\mathbb{Q}$, otherwise its fixed points $z_{0}, z_{0}^{\prime}, z_{0}^{\prime \prime}$ would individually be defined over $\left.\mathbb{Q}\right)$. Hence by the Borel and Harish-Chandra theorem (see $[\mathrm{BoH}$, Th. 9.4], though the particular case we use here is due to Ono), $\underline{T}(\mathbb{Z})$ is a lattice in $\underline{T}(\mathbb{R})$. Such a lattice contains an element of infinite order $\gamma_{0}$. The set of fixed points of $\gamma_{0}$ in $\mathbb{P}_{2}(\mathbb{C})$ is $\left\{z_{0}, z_{0}^{\prime}, z_{0}^{\prime \prime}\right\}$. Any proper nonzero linear subspace of $\mathbb{C}^{3}$ invariant under $\gamma_{0}$ is the sum of one or two geodesic lines in $\left\{z_{0}, z_{0}^{\prime}, z_{0}^{\prime \prime}\right\}$, hence is not defined over $K$ since $z_{0}$ is cubic. Therefore $\gamma_{0}$ is $K$-irreducible. As seen above, $z_{0}$ is the only fixed point of $\gamma_{0}$ in the positive cone of $q$.

Let $\gamma_{0}$ be as in Proposition 20 for $z_{0}$ given by the statement of Theorem 19. As seen in the above proof, $\gamma_{0}$ is loxodromic. Let $D^{+}$be the geodesic line in the projective model of $\mathbb{H}_{\mathbb{C}}^{2}$ with endpoints the other two Galois conjugates $z_{0}^{\prime}, z_{0}^{\prime \prime}$ of $z_{0}$ over $K$, and let $G_{D^{+}}$be its stabiliser in $G$. Up to replacing $\gamma_{0}$ by a power, we may assume that $\gamma_{0} \in G$. We may also assume that $\gamma_{0}$ is primitive in $G$, so that if $\lambda_{0}$ is its eigenvalue with modulus $>1$, then its translation length in $\mathbb{H}_{\mathbb{C}}^{2}$ is $\ln \left|\lambda_{0}\right|$ (see Equation (6)), and

$$
\operatorname{Vol}\left(G_{D^{+}} \backslash D^{+}\right)=\frac{\ln \left|\lambda_{0}\right|}{\iota_{0}}
$$

with $\iota_{0}$ defined in the statement of Theorem 19 .

Let $G_{z_{0}}$ be the stabiliser of $z_{0}$ in $G$, which is also the stabiliser of $z_{0}^{\perp}=D^{+}$, hence coincides with $G_{D^{+}}$. Let $g \in G$ be such that the geodesic line $g D^{+}$is disjoint from $\mathscr{H}_{1}$ (which is the case except for finitely many double classes in $G_{\mathscr{H}_{1}} \backslash G / G_{D^{+}}$). Let $z^{\prime}, z^{\prime \prime}$ be the endpoints of $g D^{+}$. Let $\delta_{g}$ be the common perpendicular from $\mathscr{H}_{1}$ to $g D^{+}$. The length of $\delta_{g}$ is, by [PaP2, Lem. 3.4], by Equation (9), and by the definition of the complexity,

$$
\ell\left(\delta_{g}\right)=d\left(g D^{+}, \mathscr{H}_{2}\right)-d\left(\mathscr{H}_{1}, \mathscr{H}_{2}\right)=-\ln \frac{d_{\text {Cyg }}^{\prime \prime}\left(z^{\prime}, z^{\prime \prime}\right)}{2}-\frac{\ln 2}{2}=\ln \left(\sqrt{2} c\left(g z_{0}\right)\right) .
$$

Therefore, by Corollary 13 (with $n=2$, in the case $D^{-}=\mathscr{H}_{1}$ is a horoball, whose pointwise stabiliser is trivial, and $D^{+}$is a geodesic line, with pointwise stabiliser of order $n_{0}$ as defined 
in the statement of Theorem 19, and by Equations (26), (27) and (28),

$$
\begin{aligned}
& \operatorname{Card}\left\{z \in G_{\infty} \backslash G \cdot z_{0}: c(z) \leq s\right\}=\operatorname{Card}\left\{[g] \in G_{\infty} \backslash G / G_{z_{0}}: c\left(g z_{0}\right) \leq s\right\} \\
= & \operatorname{Card}\left\{[g] \in G_{D^{-}} \backslash G / G_{D^{+}}: \ell\left(\delta_{g}\right) \leq \ln (s \sqrt{2})\right\}+\mathrm{O}(1) \\
= & \mathscr{N}_{D^{-}, D^{+}}(\ln (s \sqrt{2}))+\mathrm{O}(1)=\frac{c\left(D^{-}, D^{+}\right)}{n_{0}} e^{4 \ln (s \sqrt{2})}\left(1+\mathrm{O}\left(e^{-\kappa \ln (s \sqrt{2})}\right)\right) \\
= & \frac{32 \operatorname{Vol}\left(G_{D^{-}} \backslash D^{-}\right) \operatorname{Vol}\left(G_{D^{+}} \backslash D^{+}\right)}{9 \pi n_{0} \operatorname{Vol}\left(G \backslash \mathbb{H}_{\mathbb{C}}^{2}\right)} s^{4}\left(1+\mathrm{O}\left(s^{-\kappa}\right)\right) \\
= & \frac{256 \ln \left|\lambda_{0}\right| \zeta(3) n_{\infty}}{3 \iota_{0}\left|\mathscr{O}_{K}^{\times}\right|\left|D_{K}\right|^{3 / 2} \zeta_{K}(3) n_{0}[\bar{\Gamma}: G]} s^{4}\left(1+\mathrm{O}\left(s^{-\kappa}\right)\right) .
\end{aligned}
$$

This proves Theorem 19 .

\section{Counting arithmetic chains in hyperspherical geometry}

Let us consider again the Hermitian form $q=-z_{0} \overline{z_{2}}-z_{2} \overline{z_{0}}+\left|z_{1}\right|^{2}$ of signature $(1,2)$ on $\mathbb{C}^{3}$ with coordinates $\left(z_{0}, z_{1}, z_{2}\right)$. Following Poincaré [Poi] (who was rather using the diagonal form $-\left|z_{0}\right|^{2}+\left|z_{1}\right|^{2}+\left|z_{2}\right|^{2}$ ), see also [Car], we will call hypersphere the projective isotropic locus of $q$, that is the subspace

$$
\mathscr{H} \mathscr{S}=\left\{\left[z_{0}: z_{1}: z_{2}\right] \in \mathbb{P}_{2}(\mathbb{C}): q\left(z_{0}, z_{1}, z_{2}\right)=0\right\}
$$

of the complex projective plane $\mathbb{P}_{2}(\mathbb{C})$ with homogeneous coordinates $\left[z_{0}: z_{1}: z_{2}\right]$. It is a real analytic submanifold, diffeomorphic to the 3 -sphere $\mathbb{S}_{3}$. The subgroup $\mathrm{PSU}_{q}$ of $\mathrm{PSL}_{3}(\mathbb{C})$, acting projectively on $\mathbb{P}_{2}(\mathbb{C})$, preserves the hypersphere.

In Section 3, we introduced a natural modification $d_{\text {Cyg }}^{\prime \prime}$ of Cygan's distance $d_{\text {Cyg }}$ on $\mathscr{H} \mathscr{S}-\{\infty\}$, with $\infty=[1: 0: 0]$, as follows. We identified $\mathscr{H} \mathscr{S}-\{\infty\}$ with the real quadric (called a hyperconic by Segre) $\operatorname{Heis}_{3}=\left\{\left(w_{0}, w\right) \in \mathbb{C}^{2}: 2 \operatorname{Re} w_{0}-|w|^{2}=0\right\}$ by the map $\left(w_{0}, w\right) \mapsto\left[w_{0}: w: 1\right]$. Then $d_{\text {Cyg }}^{\prime \prime}$ is the unique map from $(\mathscr{H} \mathscr{S}-\{\infty\})^{2}$ to $[0,+\infty[$, invariant under the diagonal action of the unipotent radical of the stabiliser of $\infty$ in $\mathrm{PSU}_{q}$, such that

$$
d_{\text {Cyg }}^{\prime \prime}\left(\left(w_{0}, w\right),(0,0)\right)^{2}=\frac{|w|^{4}+4 \operatorname{Im}^{2} w_{0}}{\left(|w|^{4}+4 \operatorname{Im}^{2} w_{0}\right)^{\frac{1}{2}}+|w|^{2}}=\frac{4\left|w_{0}\right|^{2}}{2\left|w_{0}\right|+|w|^{2}} .
$$

A complex projective line $L$ in $\mathbb{P}_{2}(\mathbb{C})$ intersects the hypersphere either in the empty set, or a one point set (in which case $L$ is the unique complex projective line tangent to $\mathscr{H} \mathscr{S}$ at this point, giving at this point the canonical contact structure of $\mathscr{H} \mathscr{S}$ ), or a real analytic circle, called a chain (a notion attributed to von Staudt by [Car, footnote 3)]). A chain $C$ separates the complex projective line $L(C)$ containing it into two real discs $D_{ \pm}(C)$, which we endow with their unique Poincare metric (of constant curvature -1 ) invariant under the stabiliser of $C$ in $\mathrm{PSU}_{q}$. It is finite if and only if $C$ is a finite chain, that is, if it does not contain $\infty=[1: 0: 0]$. We refer to [Gol, §4.3] for more informations on the chains, including the following fact: the infinite chains are precisely the fibers of the vertical projection $\left(w_{0}, w\right) \mapsto w$, and the finite chains are ellipses in the affine coordinates $\left(2 \operatorname{Im} w_{0}, w\right)$ of Heis 3 whose vertical projections are circles. 


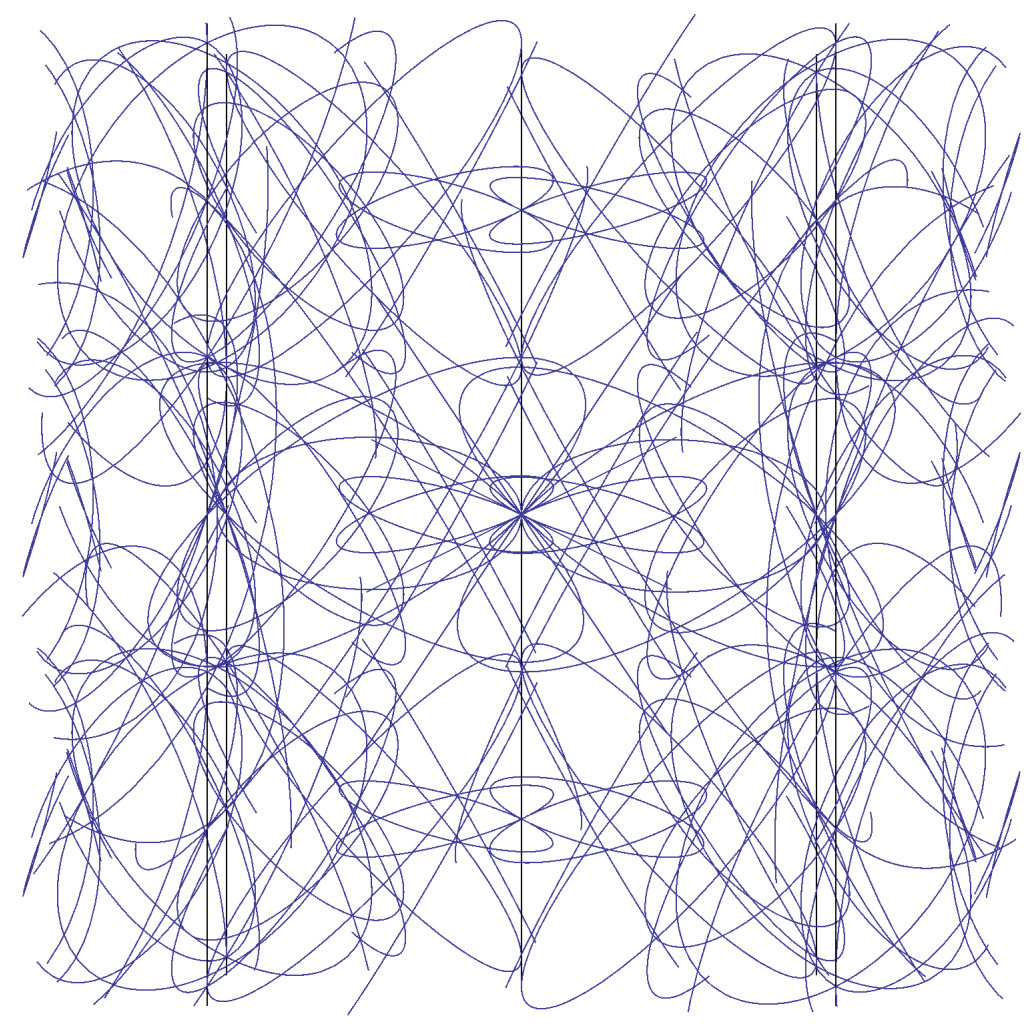

Let $K$ be an imaginary quadratic number field. For every finite index subgroup $G$ of the arithmetic lattice $\operatorname{PSU}_{q}\left(\mathscr{O}_{K}\right)$, we will denote by $G_{C}$ the stabiliser of $C$ in $G$, by $G_{\infty}$ the stabiliser of $\infty$ in $G$, and by $\operatorname{Covol}_{G}(C)$ the (common) volume of $G_{C} \backslash D_{ \pm}(C)$. A chain $C$ will be called arithmetic (over $K$ ) if $\operatorname{PSU}_{q}\left(\mathscr{O}_{K}\right)_{C}$ has a dense orbit in $C$.

Theorem 21 Let $C_{0}$ be an arithmetic chain in the hypersphere $\mathscr{H} \mathscr{S}$ over an imaginary quadratic number field $K$. Let $G$ be a finite index subgroup of $\operatorname{PSU}_{q}\left(\mathscr{O}_{K}\right)$. Then there exists a constant $\kappa>0$ such that, as $\epsilon>0$ tends to 0 , the number $\psi_{C_{0}, G}(\epsilon)$ of chains modulo $G_{\infty}$ in the $G$-orbit of $C_{0}$ with $d_{\mathrm{Cyg}}$-diameter at least $\epsilon$ is equal to

$$
\frac{2048 \zeta(3) \operatorname{Covol}_{G}\left(C_{0}\right)\left[\operatorname{PSU}_{q}\left(\mathscr{O}_{K}\right)_{\infty}: G_{\infty}\right]}{\left|\mathscr{O}_{K}^{\times}\right|\left|D_{K}\right|^{\frac{3}{2}} \zeta_{K}(3) n_{0, G}\left[\operatorname{PSU}_{q}\left(\mathscr{O}_{K}\right): G\right]} \epsilon^{-4}\left(1+\mathrm{O}\left(\epsilon^{\kappa}\right)\right)
$$

where $n_{0, G}$ is the order of the pointwise stabiliser of $C_{0}$ in $G$.

Given a complex projective line $L$ in $\mathbb{P}_{2}(\mathbb{C})$, there is a unique order 2 complex projective map with fixed point set $L$, called the reflexion in $L$. Given a finite chain $C$, contained in the projective line $L(C)$, the center of $C$ (see for instance [Gol, 4.3.3]), denoted by $\operatorname{cen}(C) \in \mathscr{H} \mathscr{S}-\{\infty\}=$ Heis $_{3}$, is the image of $\infty=[1: 0: 0]$ under the reflexion in $L(C)$. The following result is an equidistribution result in the Heisenberg group of the centers of the arithmetic chains in a given orbit under (a finite index subgroup of) $\operatorname{PSU}_{q}\left(\mathscr{O}_{K}\right)$.

Theorem 22 Let $C_{0}$ and $G$ be as in Theorem 21. As $\epsilon>0$ tends to 0 , we have

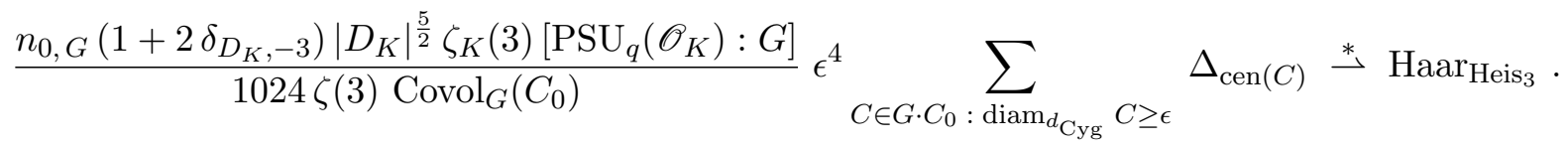


Proof of Theorem 21 and Theorem 22, As seen in Section 3, the hypersphere $\mathscr{H} \mathscr{S}$ is the boundary at infinity of the projective model of the complex hyperbolic space $\mathbb{H}_{\mathbb{C}}^{2}$. The chains are precisely the boundary at infinity of the complex geodesic lines in $\mathbb{H}_{\mathbb{C}}^{2}$. The diameter of a chain is invariant under the stabiliser in $\mathrm{PSU}_{q}$ of the horosphere $\partial \mathscr{H}_{1}$, hence is invariant under $G_{\infty}$. The counting function $\psi_{C_{0}, G}$ is thus well defined.

Recall (see for instance [Bow, in particular for the terminology) that a discrete group of isometries $\Gamma$ of a complete simply connected Riemannian manifold $M$ with sectional curvature at most -1 is geometrically finite if and only if every limit point of $\Gamma$ is either a bounded parabolic point or a conical limit point. Furthermore, the discrete groups $\Gamma$ of isometries of $M$ with finite covolume are the geometrically finite discrete groups of isometries $\Gamma$ whose limit set is the whole sphere at infinity $\partial_{\infty} M$ of $M$; then the orbit under $\Gamma$ of every point in $\partial_{\infty} M$ is dense in $\partial_{\infty} M$.

Let $C$ be a chain in $\mathscr{H} \mathscr{S}$, and let $D$ be the complex geodesic line (which is totally geodesic in $\mathbb{H}_{\mathbb{C}}^{2}$ ) with $\partial_{\infty} D=C$. Hence, $C$ is arithmetic over $K$ if and only if the stabiliser in $\operatorname{PSL}_{q}\left(\mathscr{O}_{K}\right)$ (or equivalently in $G$ ) of $D$ has finite covolume on $D$.

We denote by $D^{+}$the complex geodesic line in $\mathbb{H}_{\mathbb{C}}^{2}$ with $\partial_{\infty} D^{+}=C_{0}$. Let $G_{D^{+}}$be the stabiliser of $D^{+}$in $G$. By definition, we have

$$
\operatorname{Vol}\left(G_{D^{+}} \backslash D^{+}\right)=4 \operatorname{Covol}_{G}\left(C_{0}\right)
$$

since the sectional curvature of $D^{+}$is constant -4 and $D^{+}$has real dimension 2 .

Let $g \in G$ be such that the complex geodesic line $g D^{+}$is disjoint from $\mathscr{H}_{1}$ (which is the case except for $g$ in finitely many double classes in $\left.G_{\mathscr{H}_{1}} \backslash G / G_{D^{+}}\right)$. Let $\delta_{g}$ be the common perpendicular from $\mathscr{H}_{1}$ to $g D^{+}$. Its length $\ell\left(\delta_{g}\right)$ is the minimum of the distances from $\mathscr{H}_{1}$ to a geodesic line between two points of $\partial_{\infty}\left(g D^{+}\right)=g C_{0}$. Hence, by [PaP2, Lem. 3.4], Equation (9) and Lemma 8, we have

$$
\begin{aligned}
\ell\left(\delta_{g}\right) & =\min _{x, y \in g C_{0}, x \neq y} \ln \frac{2}{d_{\text {Cyg }}^{\prime \prime}(x, y)}-d\left(\mathscr{H}_{1}, \mathscr{H}_{2}\right)=-\max _{x, y \in g C_{0}, x \neq y} \ln \frac{d_{\mathrm{Cyg}}^{\prime \prime}(x, y)}{2}-\frac{\ln 2}{2} \\
& =-\ln \frac{\operatorname{diam}_{d_{\mathrm{Cyg}}^{\prime \prime}}\left(g C_{0}\right)}{\sqrt{2}}=-\ln \frac{\operatorname{diam}_{d_{\mathrm{Cyg}}}\left(g C_{0}\right)}{2} .
\end{aligned}
$$

Now, we apply Corollary 13 with $n=2$, in the case $D^{-}=\mathscr{H}_{1}$ is a horoball, whose pointwise stabiliser is trivial, and $D^{+}$is a complex geodesic line, whose pointwise stabiliser has order $n_{0, G}$ as defined in the statement of Theorem 21. Respectively by the definition of the counting function $\psi_{C_{0}, G}$ in the statement of Theorem 21, since the stabiliser of $C_{0}$ in $G$ is equal to $G_{D^{+}}$, by Equation (30), by Corollary 13, and by Equations (26), (27) and 
(29), we have, as $\epsilon>0$ tends to 0 ,

$$
\begin{aligned}
& \psi_{C_{0}, G}(\epsilon)=\operatorname{Card}\left\{C \in G_{\infty} \backslash G \cdot C_{0}: \operatorname{diam}_{d_{\mathrm{Cyg}}}(C) \geq \epsilon\right\} \\
& =\operatorname{Card}\left\{[g] \in G_{\infty} \backslash G / G_{D^{+}}: \operatorname{diam}_{d_{\text {Cyg }}}\left(g C_{0}\right) \geq \epsilon\right\} \\
& =\operatorname{Card}\left\{[g] \in G_{\mathscr{H}_{1}} \backslash G / G_{D^{+}}: \ell\left(\delta_{g}\right) \leq-\ln \frac{\epsilon}{2}\right\}+\mathrm{O}(1) \\
& =\mathscr{N}_{\mathscr{H}_{1}, D^{+}}\left(-\ln \frac{\epsilon}{2}\right)+\mathrm{O}(1)=\frac{c\left(\mathscr{H}_{1}, D^{+}\right)}{n_{0, G}} e^{-4 \ln \frac{\epsilon}{2}}\left(1+\mathrm{O}\left(e^{\kappa \ln \frac{\epsilon}{2}}\right)\right)
\end{aligned}
$$

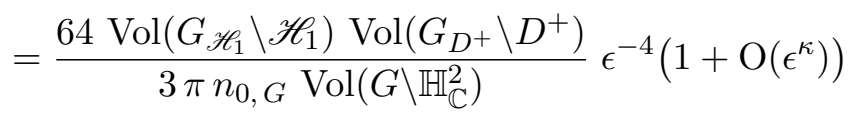

$$
\begin{aligned}
& =\frac{2048 \zeta(3) \operatorname{Covol}_{G}\left(C_{0}\right)\left[\operatorname{PSU}_{q}\left(\mathscr{O}_{K}\right)_{\infty}: G_{\infty}\right]}{\left|\mathscr{O}_{K}^{\times}\right|\left|D_{K}\right|^{\frac{3}{2}} \zeta_{K}(3) n_{0, G}\left[\operatorname{PSU}_{q}\left(\mathscr{O}_{K}\right): G\right]} \epsilon^{-4}\left(1+\mathrm{O}\left(\epsilon^{\kappa}\right)\right) .
\end{aligned}
$$

This proves Theorem 21. Let us now prove Theorem 22 .

We apply the equidistribution result in Equation (19) (in the case $D^{+}$is a complex geodesic line) of the origins or $\left(\delta_{g}\right)$ of the common perpendiculars $\delta_{g}$ from $D^{-}=\mathscr{H}_{1}$ to the images $g D^{+}$for $g \in G$. As $t \rightarrow+\infty$, we hence have

$$
\frac{3 n_{0, G} \pi \operatorname{Vol}\left(G \backslash \mathbb{H}_{\mathbb{C}}^{2}\right)}{\operatorname{Vol}\left(G_{D^{+}} \backslash D^{+}\right)} e^{-4 t} \sum_{[g] \in G / G_{D^{+}}, \ell\left(\delta_{g}\right) \leq t} \Delta_{\mathrm{or}\left(\delta_{g}\right)} \stackrel{*}{\rightarrow} \operatorname{Vol}_{\partial \mathscr{H}_{1}}
$$

Let $f: \partial_{\infty} \mathbb{H}_{\mathbb{C}}^{2}-\{\infty\}=$ Heis $_{3} \rightarrow \partial \mathscr{H}_{1}$ be the orthogonal projection map, which in horospherical coordinates is $(\zeta, u, 0) \mapsto(\zeta, u, 1)$. By Equations 15) and 20), the image of the Haar measure Haar $_{\text {Heis } 3}$ by $f$ is

$$
f_{*} \operatorname{Haar}_{\mathrm{Heis} 3}=\operatorname{vol}_{\partial \mathscr{H}_{1}} .
$$

Note that, for every chain $C$, if $r_{C}$ is the reflexion on the complex projective line containing $C$, then the geodesic line from $\infty$ to $\operatorname{cen}(C)=r_{C}(\infty)$, being invariant under $r_{C}$, is orthogonal to the complex geodesic line with boundary at infinity $C$. Hence for every $g \in G$, we have

$$
f^{-1}\left(\operatorname{or}\left(\delta_{g}\right)\right)=\operatorname{cen}\left(g C_{0}\right) .
$$

Let us use in Equation (31) the change of variables $t=-\ln \frac{\epsilon}{2}$ and the continuity of the pushforward of measures by $f^{-1}$. By Equations (26), (29) and (30), as $\epsilon>0$ tends to 0 , the measures

$$
\frac{n_{0, G}\left(1+2 \delta_{D_{K},-3}\right)\left|D_{K}\right|^{\frac{5}{2}} \zeta_{K}(3)\left[\operatorname{PSU}_{q}\left(\mathscr{O}_{K}\right): G\right]}{1024 \zeta(3) \operatorname{Covol}_{G}\left(C_{0}\right)} \epsilon^{4} \sum_{[g] \in G / G_{D^{+}}} \sum_{\operatorname{diam}_{d_{\mathrm{Cyg}}}\left(g C_{0}\right) \geq \epsilon} \Delta_{\operatorname{cen}\left(g C_{0}\right)}
$$

weak-star converge to the Haar measure $\mathrm{Haar}_{\mathrm{Heis}_{3}}$. This proves Theorem 22.

Example. Let $K=\mathbb{Q}(i)$, so that $\left|D_{K}\right|=\left|\mathscr{O}_{K}^{\times}\right|=4$. Let $C_{0}$ be the intersection of the hypersphere and the complex projective line $L\left(C_{0}\right)$ with equation $z_{1}=0$.

Let us use $\infty=[1: 0: 0]$ as the point at infinity in $L\left(C_{0}\right)$, and let us identify $L\left(C_{0}\right)$ with $\mathbb{P}_{1}(\mathbb{C})=\mathbb{C} \cup\{\infty\}$ by the map $\left[z_{0}: 0: z_{2}\right] \mapsto z=\frac{z_{0}}{z_{2}}$. Then the restriction $\Gamma_{1,1}$ to $L\left(C_{0}\right)$ of the stabiliser in $\operatorname{PSU}_{q}\left(\mathscr{O}_{K}\right)$ of $C_{0}$ is exactly the subgroup of $\mathrm{PSL}_{2}(\mathbb{C})$ preserving the right 
halfplane with equation $\operatorname{Re} z>0$. The homography $z \mapsto i z$ sends the right halfplane to the upper halfplane $\mathbb{H}_{\mathbb{R}}^{2}$. Hence $\left(\begin{array}{ll}i & 0 \\ 0 & 1\end{array}\right) \Gamma_{1,1}\left(\begin{array}{cc}-i & 0 \\ 0 & 1\end{array}\right)$ is the subgroup of $\operatorname{PSL}_{2}(\mathbb{C})$ preserving $\mathbb{H}_{\mathbb{R}}^{2}$ and having coefficients in $\mathbb{Z}[i]$. It is hence equal to $\operatorname{PSL}_{2}(\mathbb{Z})$, and it is well known that the hyperbolic volume of $\mathrm{PSL}_{2}(\mathbb{Z}) \backslash \mathbb{H}_{\mathbb{R}}^{2}$ is $\frac{\pi}{3}$. In particular, $C_{0}$ is an arithmetic chain over $K$.

Let $\alpha$ be an element in the pointwise stabiliser of $C_{0}$ in $\operatorname{PSU}_{q}\left(\mathscr{O}_{K}\right)$. Then $\alpha$ fixes $\infty=[1: 0: 0]$ and $[0: 0: 1]$, hence consists of diagonal matrices. The diagonal coefficients $\lambda_{1}, \lambda_{2}, \lambda_{3}$ of $\alpha$ belong to $\mathbb{Z}[i]^{\times}=\{ \pm 1, \pm i\}$. We have $\lambda_{1}=\lambda_{3}$, otherwise $\alpha$ would not act by the identity on $C_{0}$. Therefore $\left(\lambda_{1}, \lambda_{2}, \lambda_{3}\right)$ belongs to $\{(1,1,1),(-1,1,-1),(i,-1, i)$, $(-i,-1,-i)\}$. This gives the following values

$$
\operatorname{Covol}_{\mathrm{PSU}_{q}(\mathbb{Z}[i])}\left(C_{0}\right)=\frac{\pi}{3} \text { and } n_{0, \mathrm{PSU}_{q}(\mathbb{Z}[i])}=4 .
$$

Theorems 21 and 22 then give

$$
\psi_{C_{0}, \mathrm{PSU}_{q}(\mathbb{Z}[i])}(\epsilon)=\frac{16 \pi \zeta(3)}{3 \zeta_{\mathbb{Q}(i)}(3)} \epsilon^{-4}\left(1+\mathrm{O}\left(\epsilon^{\kappa}\right)\right),
$$

and

$$
\frac{3 \zeta_{\mathbb{Q}(i)}(3)}{4 \pi \zeta(3)} \epsilon^{4} \sum_{C \in \mathrm{PSU}_{q}(\mathbb{Z}[i]) \cdot C_{0}: \operatorname{diam}_{d_{\mathrm{Cyg}}} C \geq \epsilon} \Delta_{\mathrm{cen}(C)} \stackrel{*}{\rightarrow} \mathrm{Haar}_{\mathrm{Heis} 3} .
$$

The above picture, as well as the one in the introduction, represents the orbit of $C_{0}$ under the arithmetic lattice $\mathrm{PSU}_{q}(\mathbb{Z}[i])$. The pictures were produced using Mathematica and they show images of the chain $C_{0}$ by elements of $\operatorname{PSU}_{q}(\mathbb{Z}[i])$ of word length at most 16 in the generating set given in [FaFP, Theo. 7]. Both images show the same set of chains from two different viewpoints in the 3-dimensional space Heis 3 , except that in the second one we removed some of the smaller chains in order to make the structure more visible.

\section{References}

[BeQ] Y. Benoist and J.-F. Quint. Stationary measures and invariant subsets of homogeneous spaces II. J. Amer. Math. Soc. 26 (2013) 659-734.

[BF] M. Björklund and A. Fish. Equidistribution of dilations of polynomial curves in nilmanifolds. Proc. Amer. Math. Soc. 137 (2009) 2111-2123.

[BoH] A. Borel and Harish-Chandra. Arithmetic subgroups of algebraic groups. Ann. of Math. 75 (1962) 485-535.

[Bow] B. Bowditch. Geometrical finiteness with variable negative curvature. Duke Math. J. 77 (1995) 229-274.

[Bre] E. Breuillard. Local limit theorems and equidistribution of random walks on the Heisenberg group. Geom. Funct. Anal. 15 (2005) 35-82.

[BrH] M. R. Bridson and A. Haefliger. Metric spaces of non-positive curvature. Grund. math. Wiss. 319, Springer Verlag, 1999.

[Car] É. Cartan. Sur le groupe de la géométrie hypersphérique. Comment. Math. Helv. 4 (1932) 158-171. 
[CI] K. Corlette and A. Iozzi. Limit sets of discrete groups of isometries of exotic hyperbolic spaces. Trans. Amer. Math. Soc. 351 (1999) 1507-1530.

[Cyg] J. Cygan. Wiener's test for Brownian motion on the Heisenberg group. Coll. Math. 39 (1978) 367-373.

[EiW] M. Einsiedler and T. Ward. Ergodic theory with a view towards number theory. Grad. Texts Math. 259, Springer-Verlag, 2011.

[EmS] V. Emery and M. Stover. Covolumes of nonuniform lattices in PU(n,1). Amer. J. Math. 136 (2014) 143-164.

[FaFP] E. Falbel, G. Francsics, and J. Parker. The geometry of the Gauss-Picard modular group. Math. Ann. 349 (2011) 459-508.

[FaP] E. Falbel and J. Parker. The geometry of the Eisenstein-Picard modular group. Duke Math. J. 131 (2006) 249-289.

[Feu] J.-M. Feustel. Über die Spitzen von Modulflächen zur zweidimensionalen komplexen Einheitskugel. Preprint Series 13. Akad.Wiss. DDR, ZIMM, Berlin, 14, 1977.

[FS] G. B. Folland and E. M. Stein. Estimates for the $\bar{\partial}_{b}$ complex and analysis on the Heisenberg group. Comm. Pure Appl. Math. 27 (1974) 429-522.

[Gol] W. M. Goldman. Complex hyperbolic geometry. Oxford Univ. Press, 1999.

[GT] B. Green and T. Tao. The quantitative behaviour of polynomial orbits on nilmanifolds. Ann. of Math. 175 (2012) 465-540.

[HaW] G. H. Hardy and E. M. Wright. An introduction to the theory of numbers. Oxford Univ. Press, sixth ed., 2008.

[HeP1] S. Hersonsky and F. Paulin. Counting orbit points in coverings of negatively curved manifolds and Hausdorff dimension of cusp excursions. Erg. Theo. Dyn. Sys. 24 (2004) 803-824.

[HeP2] S. Hersonsky and F. Paulin. On the volumes of complex hyperbolic manifolds. Duke Math. J. 84 (1996) 719-737.

[Hol1] R.-P. Holzapfel. Arithmetische Kugelquotientenflächen I/II. Sem. Ber. Math. Humb. Univ. Berlin, 14, 1978.

[Hol2] R.-P. Holzapfel. Ball and surface arithmetics. Asp. Math. E29, Vieweg, 1998.

[Hol3] R.-P. Holzapfel. Volumes of fundamental domains of Picard modular groups. In "Arithmetic of complex manifolds" (Erlangen, 1988), 60-88, Lecture Notes Math. 1399, Springer Verlag 1989.

[Kim] I. Kim. Counting, mixing and equidistribution of horospheres in geometrically finite rank one locally symmetric manifolds. J. reine angew. Math. 704 (2015) 85-133.

[KK] I. Kim and J. Kim. On the volumes of canonical cusps of complex hyperbolic manifolds. J. Korean Math. Soc. 46 (2009) 513-521.

[Kor] A. Korányi. Geometric properties of Heisenberg-type groups. Adv. Math. 56 (1985) 28-38.

[Kra] S. Krantz. Explorations in harmonic analysis, with applications to complex function theory and the Heisenberg group. Appl. Num. Harm. Anal., Birkhäuser, 2009.

[Mos] G. D. Mostow. Strong rigidity of locally symetric spaces. Ann. Math. Studies 78, Princeton Univ. Press, 1973.

[OhS1] H. Oh and N. Shah. The asymptotic distribution of circles in the orbits of Kleinian groups. Invent. Math. 187 (2012) 1-35. 
[OhS2] H. Oh and N. Shah. Counting visible circles on the sphere and Kleinian groups. Preprint [arXiv: 1004.2129], to appear in "Geometry, Topology and Dynamics in Negative Curvature" (ICM 2010 satellite conference, Bangalore), C. S. Aravinda, T. Farrell, J.-F. Lafont eds, London Math. Soc. Lect. Notes 425, Cambridge Univ. Press 2016.

[OP] J.-P. Otal and M. Peigné. Principe variationnel et groupes kleiniens. Duke Math. J. 125 (2004) 15-44.

[Par1] J. Parker. Shimizu's lemma for complex hyperbolic space. Inter. J. Math. 3 (1992) 291-308.

[Par2] J. Parker. On the volumes of cusped, complex hyperbolic manifolds and orbifolds. Duke Math. J. 94 (1998) 433-464.

[Par3] J. Parker. Hyperbolic spaces. Jyväskylä Lect. Math. 2 (2008), http://urn.fi/URN:ISBN:95139-3132-2 .

[Par4] J. Parker. Traces in complex hyperbolic geometry. In "Geometry, Topology and Dynamics of Character Varieties", Lect. Notes Ser. 23, Inst. Math. Sciences, Nat. Univ. Singapore, 191-245. World Scientific, 2012.

[PaP1] J. Parkkonen and F. Paulin. Prescribing the behaviour of geodesics in negative curvature. Geom. \& Topo. 14 (2010) 277-392.

[PaP2] J. Parkkonen and F. Paulin. Spiraling spectra of geodesic lines in negatively curved manifolds. Math. Z. 268 (2011) 101-142.

[PaP3] J. Parkkonen and F. Paulin. Skinning measure in negative curvature and equidistribution of equidistant submanifolds. Erg. Theo. Dyn. Sys. 34 (2014) 1310-1342.

[PaP4] J. Parkkonen and F. Paulin. On the arithmetic of crossratios and generalised Mertens' formulas. Special issue "Aux croisements de la géométrie hyperbolique et de l'arithmétique", F. Dal'Bo, C. Lecuire eds, Ann. Fac. Sci. Toulouse 23 (2014) 967-1022.

[PaP5] J. Parkkonen and F. Paulin. On the hyperbolic orbital counting problem in conjugacy classes. Math. Z. 279 (2015) 1175-1196.

[PaP6] J. Parkkonen and F. Paulin. Counting arcs in negative curvature. Preprint [arXiv:1203. 0175], to appear in "Geometry, Topology and Dynamics in Negative Curvature" (ICM 2010 satellite conference, Bangalore), C. S. Aravinda, T. Farrell, J.-F. Lafont eds, London Math. Soc. Lect. Notes 425, Cambridge Univ. Press, 2016.

[PaP7] J. Parkkonen and F. Paulin. Counting common perpendicular arcs in negative curvature. Preprint [arXiv:1305.1332], to appear in Erg. Theo. Dyn. Syst.

[Poi] H. Poincaré. Les fonctions analytiques de deux variables et la représentation conforme. Rendic. Circ. Mat. Palermo 23 (1907) 207-212.

[Rob] T. Roblin. Ergodicité et équidistribution en courbure négative. Mémoire Soc. Math. France, 95 (2003).

[Sch] W. Scharlau. Quadratic and Hermitian forms. Grund. math. Wiss. 270, Springer-Verlag, 1985.

[Sto] M. Stover. Volumes of Picard modular surfaces. Proc. Amer. Math. Soc. 139 (2011) 30453056.

[Wal] A. Walfisz. Weylsche Exponentialsummen in der neueren Zahlentheorie. VEB Deutscher Verlag der Wissenschaften, 1963.

[Zin] T. Zink. Über die Anzahl der Spitzen einiger arithmetischer Untergruppen unitärer Gruppen. Math. Nachr. 89 (1979) 315-320. 
Department of Mathematics and Statistics, P.O. Box 35 40014 University of Jyväskylä, FINLAND.

e-mail: jouni.t.parkkonen@jyu.fi

Laboratoire de mathématique d'Orsay, Univ. Paris-Sud, CNRS

Université Paris-Saclay, 91405 ORSAY Cedex, FRANCE

e-mail: frederic.paulin@math.u-psud.fr 